\title{
Summer outbursts in the coma of comet 67P/Churyumov-Gerasimenko as observed by Rosetta-VIRTIS
}

\author{
G. Rinaldi, ${ }^{1,2 \star}$ D. Bockelée-Morvan, ${ }^{3 \star}$ M. Ciarniello, ${ }^{1}$ G. P. Tozzi, ${ }^{4}$ F. Capaccioni, ${ }^{1 \star}$ \\ S. L. Ivanovski, ${ }^{1,2}$ G. Filacchione, ${ }^{1}$ U. Fink,${ }^{5}$ L. Doose,${ }^{5}$ F. Taylor,${ }^{6}$ D. Kappel, ${ }^{7}$ \\ S. Erard, ${ }^{3}$ C. Leyrat, ${ }^{3}$ A. Raponi, ${ }^{1}$ E. D’ Aversa, ${ }^{1}$ M. T. Capria, ${ }^{1}$ A. Longobardo,,${ }^{1,2}$ \\ E. Palomba, ${ }^{1}$ F. Tosi, ${ }^{1}$ A. Migliorini, ${ }^{1}$ A. Rotundi, ${ }^{1,2}$ V. Della Corte ${ }^{1,2}$ and M. Salatti ${ }^{8}$ \\ ${ }^{1}$ Istituto di Astrofisica e Planetologia Spaziali, Istituto Nazionale di Astrofisica, via del Fosso del Cavaliere 10000133 Rome, Italy \\ ${ }^{2}$ Universita' degli Studi di Napoli Parthenope, Dip. di Scienze e Tecnologie, CDN IC4, I-80143 Naples, Italy \\ ${ }^{3}$ LESIA, Observatoire de Paris, Universitè PSL,CNRS,Sorbonne Universitè, Univ. Paris Diderot Sorbonne Paris Citè F-92195 Meudon, France \\ ${ }^{4}$ INAF - Osservatorio Astrofisico di Arcetri , via LargoEnrico Fermi, 50125, Firenze, Italy \\ ${ }^{5}$ Lunar and Planetary Laboratory, University of Arizona, Tucson, AZ 85721, USA \\ ${ }^{6}$ Departement of Physics, Oxford University, Parks Rd, OX13PJ, Regno Unito, Oxford, UK \\ ${ }^{7}$ Institute of Planetary Research, DLR, D-12489 Berlin, Germany \\ ${ }^{8}$ ASI, Via del Politecnico snc, I-00133 Rome, Italy
}

Accepted 2018 August 16. Received 2018 August 16; in original form 2018 March 12

\begin{abstract}
We present an analysis of transient events observed by the Visible InfraRed Thermal Imaging Spectrometer, instrument aboard Rosetta, for the dates of 2015 August 10, September 13 and 14, during the two months surrounding the comet perihelion passage of the Rosetta spacecraft. We detected and characterized events with life-times ranging from 26 min down to 6 min. The temporal evolution of the outburst shows a sudden increase of radiance from quiescent coma to the maximum in a few minutes. This rapid onset is correlated with a change of the visible dust colour from red, $15-18 \pm 3$ per cent/100 nm, to bluer with values of 7-10 \pm 0.3 per cent/100 nm. The dust morphology of these outbursts can be classified into two main types: narrow and collimated plumes (August 10, September 13) and broad blobs (September 14). The observations suggest that there are localized regions on the surface that are more prone to outbursts than the rest of the nucleus. The projected dust velocity during the outburst events ranges between $22.2 \pm 2.2 \mathrm{~m} \mathrm{~s}^{-1}$ and $64.9 \pm 10.6 \mathrm{~m} \mathrm{~s}^{-1}$. The total ejected mass during an outburst event is estimated to be between 10 and 500 tons for a duration of 6-26 min assuming size distribution indices between -2.5 and -3 .
\end{abstract}

Key words: methods: data analysis - methods:observational - space vehicles: instruments techniques: imaging spectroscopy - comets: individual: 67P/Churyumov-Gerasimenko.

\section{INTRODUCTION}

The primary objective of this work is to analyse several outbursts of gas and dust from the comet 67P/Churyumov-Gerasimenko (hereafter $67 \mathrm{P} / \mathrm{CG}$ ) and to investigate the morphology and other properties of the plumes ejected during these events. The Rosetta probe, orbiting close to the comet 67P/CG observed frequent outbursts with an unprecedented level of detail unlike those of previous studies (A'Hearn et al. 2005; Farnham et al. 2007; Belton et al. 2008).

\footnotetext{
^E-mail: Giovanna.rinaldi@iaps.inaf.it (G.R.);

Dominique.Bockelee@obspm.fr (D.B.-M.);

fabrizio.capaccioni@iaps.inaf.it (F.C.)
}

These outbursts are characterized by a sudden and short increase of the dust emission, from localized areas (Vincent et al. 2016) with variable degree of collimation, followed by a gradual decrease of activity.

From 2014 to 2016, several outbursts were observed by Rosetta (Tubiana et al. 2015; Grün et al. 2016; Knollenberg et al. 2016; Vincent et al. 2016; Agarwal et al. 2017; Della Corte et al. 2017). These observations provided a detailed characterization of the nature and evolution of 67P/CG outbursts and determined, in a few cases, their periodicities.

Tubiana et al. (2015) reported a small outburst on 2014 April 30, which was observed by the Narrow Angle Camera (NAC) of the Optical, Spectroscopic, and Infrared Remote Imaging System (OSIRIS), during the approach of Rosetta to perihelion. 
On 2015 March 12, Knollenberg et al. (2016) observed, with the OSIRIS Wide Angle Camera (WAC), a small outburst originating from the Imhotep region at the foot of the big lobe of 67P/CG. The source region was on the nightside of the nucleus, implying activity from a non-illuminated area. The outburst lasted less than $1 \mathrm{~h}$, and the average dust production rate grew rapidly in the initial $4 \mathrm{~min}$ up to $1 \mathrm{kgs}^{-1}$ and remained constant until the end. The differential dust size distribution was described by a power law with index of about -2.6 implying a dust-to-gas ratio in the outburst ejecta in the range $0.6-1.8$.

In 2015 August, during the perihelion passage, Vincent et al. (2016) detected and characterized 34 outbursts with the Rosetta cameras, one every 2.4 nucleus rotations. They identified three main dust plume morphologies: a narrow jet and a broad fan, plus more complex plumes featuring both of these types together. They found a correlation between the outbursts sources on the nucleus with morphological boundaries; the sources regions were often identified in the proximity of steep scarps or cliffs.

On 2016 February 19, six months after perihelion, nine Rosetta instruments observed an outburst of gas and dust from the nucleus of 67P/CG (Grün et al. 2016). The likely outburst source was identified in the region called Atum, where Vincent et al. (2016) observed two other outbursts during perihelion. Looking at OSIRIS images the outburst appears as a cloud, consistent with the relative long duration of the dust impact phase as measured by the Grain Impact Analyzer and Dust Accumulator (GIADA) instrument, indicating that the emitted dust cone was very broad. Grün et al. (2016) interpreted the event as triggered by thermal stresses, fracture mechanics, and gravity in the form of landslides or avalanches.

Pajola et al. (2017) studied morphological changes associated with dusty outbursts that appear to result from cliff collapse, revealing fresh icy regions with an albedo at least six times higher than the rest of the surface of the nucleus.

On 2016 July 3, several instruments onboard Rosetta detected an outburst when 67P/CG was at 3.32 au from the Sun after perihelion. Agarwal et al. (2017) reported the inferred properties of the ejected dust, and that the surface change at the site of the outburst occurred at local sunrise, lasting $14-68 \mathrm{~min}$. The outburst left a $10 \mathrm{~m}$-sized icy patch on the nucleus surface. The ejected material included refractory grains of several hundred microns in size, and submicronsized water ice grains.

Della Corte et al. (2017) presented observations of an outburst event that occurred on 2016 September 5 a few days before the final landing of Rosetta on the surface of 67P/CG. GIADA observed the temporal and spatial variation of particles from submicron to millimetre from a distance less than $5 \mathrm{~km}$ from the comet surface.

In this paper, we present an analysis of the outbursts observed by Visible InfraRed Thermal Imaging Spectrometer (VIRTIS) on 2015 August 10 and September 13 and 14, when the comet was at heliocentric distances of 1.25-1.30 au, two days before and one month after the perihelion passage, respectively. The spectra acquired by the VIRTIS-H (VH) channel allowed us to infer the presence of submicrometric particles in the ejecta of the September 13 and 14 outbursts to interpret the blue colours and high colour temperatures measured in the infrared (Bockelée-Morvan et al. 2017). In this paper, we use the VIRTIS-M (VM) observations (Section 2) and combine them with the $\mathrm{VH}$ data published in Bockelée-Morvan et al. (2017). The dust properties of the outburst are discussed in terms of spatial distribution, temporal evolution (Section 3), and location (Section 4). We also analysed dust physical properties such as particle velocity (Section 5), reflectance and colour (Section 6), fill- ing factor (Section 7), and dust mass loss (Section 8). Conclusions and future perspectives are provided in Section 9.

\section{OBSERVATIONS AND DATA REDUCTION}

The VIRTIS onboard Rosetta is composed of two experiments: VH (Drossart et al. 2000), a cross-dispersing single-point spectrometer providing spectra with high spectral resolution in the range 1.9-5 $\mu \mathrm{m}$, and VM (Coradini et al. 2007), an imaging spectrometer working in the range between 0.25 and $5 \mu \mathrm{m}$ by with two channels: the visible (VIS) and the infrared (IR) covering the $0.25-1 \mu \mathrm{m}$ and the 1-5 $\mu \mathrm{m}$ spectral intervals, respectively. VM is a slit spectrometer, and thus the instantaneous field of view (FOV) covers a single line on the target. The VM spectral images are acquired sequentially by scanning the FOV from the top to the bottom for a given number of lines (see Table 1). This means any scanned image is obtained over a duration that depends on the number of lines and the exposure time for each line (Coradini et al. 2007).

In this paper, we consider three sets of VM observations, showing outburst ejecta acquired on 2015 August 10, September 13 and September 14, i.e. a few days before and approximately one month after the perihelion passage at heliocentric distances from 1.2 to $1.3 \mathrm{au}$. Due to the failure of the IR channel in early 2015 May, our analysis is restricted to only VIS hyperspectral images. A list of the observations with all the relevant information is provided in Table 1. The geometry information for each VM observation is calculated by a SPICE routine (Acton 1996) using the spacecraft's trajectory/orientation reconstructed kernels and the 67P/CG SHAP5 shape model for the comet nucleus (Jorda et al. 2016).

The first data set we analysed is shown in Fig. 1 A. This was obtained on 2015 August 10 when the spacecraft was at a distance from the comet nucleus of $309 \mathrm{~km}$, corresponding to an FOV of $18 \mathrm{~km}$ in the horizontal direction and $9 \mathrm{~km}$ in the vertical one. The spacecraft was approximately in a terminator orbit, so that the sun illuminated only one hemisphere of the nucleus, while the other one was in darkness.

On 2015 September 13, from 6:50 to 23:32 h UT, VM acquired 25 images that lasted about $30 \mathrm{~min}$ each. Among them, we analysed a subset of three images (Fig. 2 B, C, and D) obtained from a target distance of $314 \mathrm{~km}$ with the FOV covering an area of $20 \times 17 \mathrm{~km}$ at that distance. On this date, we observed a strong, anomalous activity of the coma starting between 13:25 and 15:00 h. The instrument detected one large outburst and two smaller outbursts about $10 \mathrm{~min}$ later. In this paper, we define outbursts as strong, big, small, or mini according to their brightness level at the maximum of the event and their lifetime.

The third data set of this paper, two image cubes acquired on 2015 September 14, shows one mini outburst followed by a strong outburst about 10 min later (Fig. 3). For this data set, the FOV of the $\mathrm{VM}$ instrument is observing only the coma above the illuminated nucleus, spanning about 18 and $1.5 \mathrm{~km}$ in the horizontal and vertical direction, respectively, referred to a target distance of $316 \mathrm{~km}$.

The spectra and images used for our analysis were reduced using the VIRTIS reduction pipeline (Ammannito et al. 2006; Filacchione et al. 2006) and additional corrections derived from in-flight data. Moreover, we remove defective pixels or cosmic ray strikes using a median filter de-spiking algorithm, which is employed only along the spatial dimensions of the data, leaving the spectral data intact.

As a measure of the continuum intensity in the VIS, we chose a narrow band pass of $0.10 \mu \mathrm{m}$ width centred at $0.55 \mu \mathrm{m}$, while in the IR we selected two spectral regions at 2.42 and $3.85 \mu \mathrm{m}$. All IR continuum radiances were averaged over 50 spectra. 
Table 1. VM observations analysed in this work.

\begin{tabular}{|c|c|c|c|c|c|c|c|c|c|c|c|}
\hline \multirow[t]{2}{*}{ Img } & VIS file name & $\begin{array}{c}\text { Cube } \\
\text { dimension }\end{array}$ & $\begin{array}{l}\text { Start } \\
\text { time } \\
\text { (UT) }\end{array}$ & $\begin{array}{c}\text { Duration } \\
\text { time } \\
\text { (s) }\end{array}$ & $\begin{array}{c}\text { Exp } \\
\text { time } \\
(\mathrm{s})\end{array}$ & $\begin{array}{c}\mathrm{S} / \mathrm{C} \\
\text { distance } \\
(\mathrm{km})\end{array}$ & $\begin{array}{c}\text { Spatial } \\
\text { resolution } \\
\left(\mathrm{m} \mathrm{px}^{-1}\right)\end{array}$ & $\begin{array}{l}\text { Phase } \\
\text { angle } \\
(0)\end{array}$ & $\begin{array}{c}\text { Sub/Solar } \\
\text { Lat } \\
\text { (o) }\end{array}$ & $\begin{array}{c}\text { Sub/Solar } \\
\text { Long } \\
\text { (०) }\end{array}$ & $\begin{array}{l}\mathrm{r}_{\mathrm{h}} \\
(\mathrm{au})\end{array}$ \\
\hline & \multicolumn{11}{|c|}{ DATA OBSERVATIONS } \\
\hline & & & & & 2015 & & & & & & \\
\hline \multirow[t]{2}{*}{ A } & V1_00397784631 & 256129432 & $23: 45: 09$ & 2700 & 16 & 309 & 77 & 89 & -25.2 & 155.1 & 1.25 \\
\hline & \multicolumn{11}{|c|}{2015 Sept 13} \\
\hline B & V1_00400771480 & 25698432 & $13: 59: 18$ & 1980 & 16 & 314 & 78 & 108 & -29.42 & 308.8 & 1.30 \\
\hline $\mathrm{C}$ & V1_00400773580 & 25698432 & $14: 34: 18$ & 1980 & 16 & 314 & 78 & 109 & -32.27 & 285.3 & 1.30 \\
\hline \multirow[t]{2}{*}{$\mathrm{D}$} & V1_00400775680 & 25698432 & 15:09:18 & 1980 & 16 & 314 & 78 & 109 & -32.51 & 258.5 & 1.30 \\
\hline & \multicolumn{11}{|c|}{2015 Sept 14} \\
\hline $\mathrm{E}$ & V1_00400875328 & 25626432 & $18: 16: 47$ & 1640 & 35 & 316 & 79 & 98.84 & -22.28 & 173.3 & 1.30 \\
\hline $\mathrm{F}$ & V1_00400877229 & 25620432 & $18: 48: 28$ & 438 & 16 & 316 & 79 & 98.71 & -22.91 & 166.2 & 1.30 \\
\hline
\end{tabular}

Notes. Img: Assigned letter for each image cube.

VIS file name: Observation file names.

Cube dimension: Cube size in number of samples (256 fixed pixel number), number of scan lines, and spectral bands (432 for each channel).

Start time: Start time of each image cube (UT).

Duration time: Total duration time for each image cube from acquisition start to stop.

Exp time: Exposure time for each line.

S/C distance: Distance from the comet centre.

Spatial resolution: Pixel size at the distance of each observation.

Phase angle: Observation phase angle.

Sub/Solat Lat: Subsolar Latitude.

Sub/Solat Lon: Subsolar Longitude.

$\mathrm{r}_{\mathrm{h}}$ : Heliocentric distance.

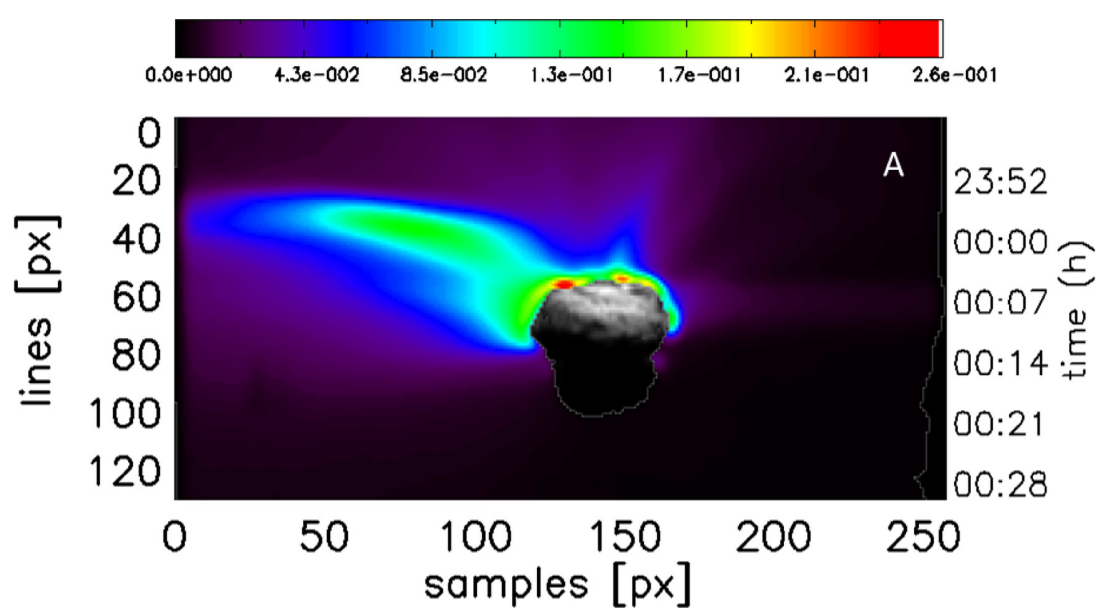

Figure 1. Radiance at $0.55 \mu \mathrm{m}$ for image V1_00397784631 (A), acquired on 2015 August 10, showing the nucleus, dust coma, and outburst ejecta (blob on the left). The image details are listed in Table 1. A VM image of the nucleus at $0.55 \mu \mathrm{m}$ is superimposed for better visualization. The radiance has units of W $\mathrm{m}^{-2} \mathrm{sr}^{-1} \mu \mathrm{m}^{-1}$.

\section{OUTBURST MORPHOLOGY AND LIGHT CURVES}

The goal of this section is to analyse the evolution of $67 \mathrm{P} / \mathrm{CG}$ outbursts and characterize the spatial distribution of both the dust background and the ejected dust. In particular, VM observation sequences acquired during outburst activity allow us studying the morphological and dynamic changes of the ejected dust for different types of outbursts.

We identified two main dust plume morphologies associated with these events: narrow jets (August 10 and September 13) and broad plumes (September 14). Vincent et al. (2016) distinguished three type of outburst morphologies: Type A is associated with a very collimated jet that expands beyond the FOV,
Type $\mathrm{B}$ is a broad plume, or wide dust fan, and Type $\mathrm{C}$ is a complex event, often combining both a narrow and a broad feature.

In general, we know that the lifetime of these events ranges between 5 and $30 \mathrm{~min}$ (Knollenberg et al. 2016; Vincent et al. 2016) due to the VM acquisition time and FOV size, we do not know if the image cubes were acquired shortly after the outburst started or later, and if most of the material is still in the frame. The comparison between the light curves of VH (Bockelée-Morvan et al. 2017) and the temporal profiles of VM (Figs 4 and 5) help us to understand what stage of the event we are observing and to infer the dynamic behaviour of the outburst (Section 5).

In Table 2, we list all the relevant information obtained from the analysis of our data set as outburst size or strength, time, duration, 

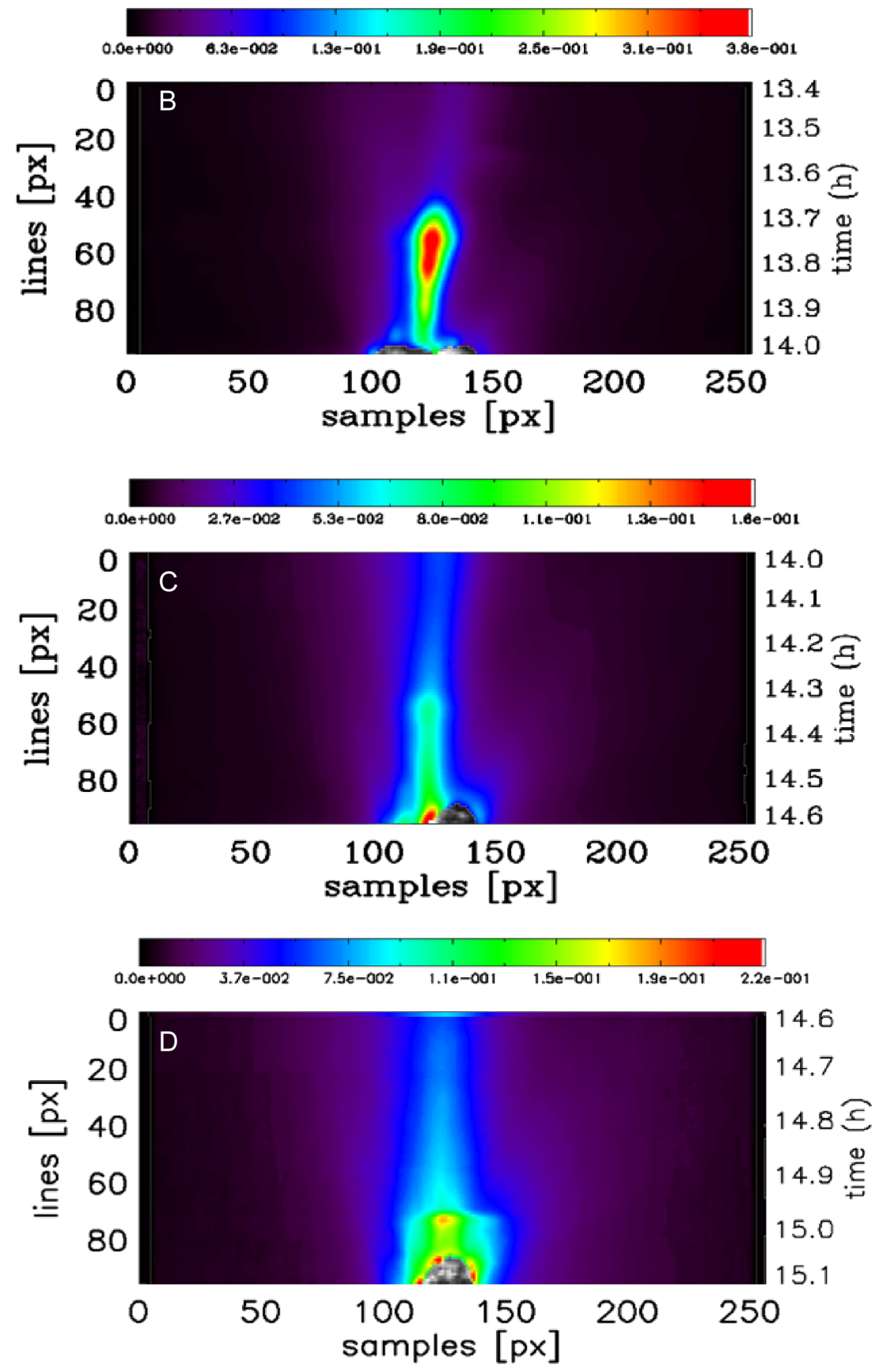

Figure 2. Radiance value at $0.55 \mu \mathrm{m}$ for the images V1_00400771480 (B), V1_00400773580 (C), and V1_00400775680 (D) acquired on 2015 September 13 , showing the nucleus, dust coma, and outburst ejecta (collimated ejecta). A VM image of the nucleus at $0.55 \mu \mathrm{m}$ is superimposed for better visualization. The radiance has units of $\mathrm{W} \mathrm{m}^{-2} \mathrm{sr}^{-1} \mu \mathrm{m}^{-1}$.

longitude, and latitude of the estimated source region of the outburst (see Section 4), radiance level, and colour at the maximum of the light curves (see discussion in Section 6). The duration is computed using the time at which the radiance returned to the pre-outburst value according to the $\mathrm{VH}$ observations (Bockelée-Morvan et al. 2017) and the strength or outburst size is defined in terms of radiance level at the maximum of the event (eighth column). 'Big' outbursts are events with a radiance level higher than $0.1 \mathrm{~W} \mathrm{~m}^{-2} \mathrm{sr}^{-1} \mu \mathrm{m}^{-1}$ and 'mini' with a radiance level less than $0.1 \mathrm{~W} \mathrm{~m}^{-2} \mathrm{sr}^{-1} \mu \mathrm{m}^{-1}$. The outburst (f) is defined as 'Strong' because it is the strongest event observed on 67P/CG by VIRTIS and other instruments with a radiance level of $1 \mathrm{~W} \mathrm{~m}^{-2} \mathrm{sr}^{-1} \mu \mathrm{m}^{-1}$, similar to the typical radiance values measured on illuminated regions of the nucleus surface in the VIS channel (Section 3.3 and El-Maarry et al. 2017; Vincent et al. 2017).

\subsection{The August 10 outburst}

Fig. 1 shows the outburst observed on August 10 starting at 23:50 h and lasting until 00:07 h. The hyperspectral cube was acquired in 


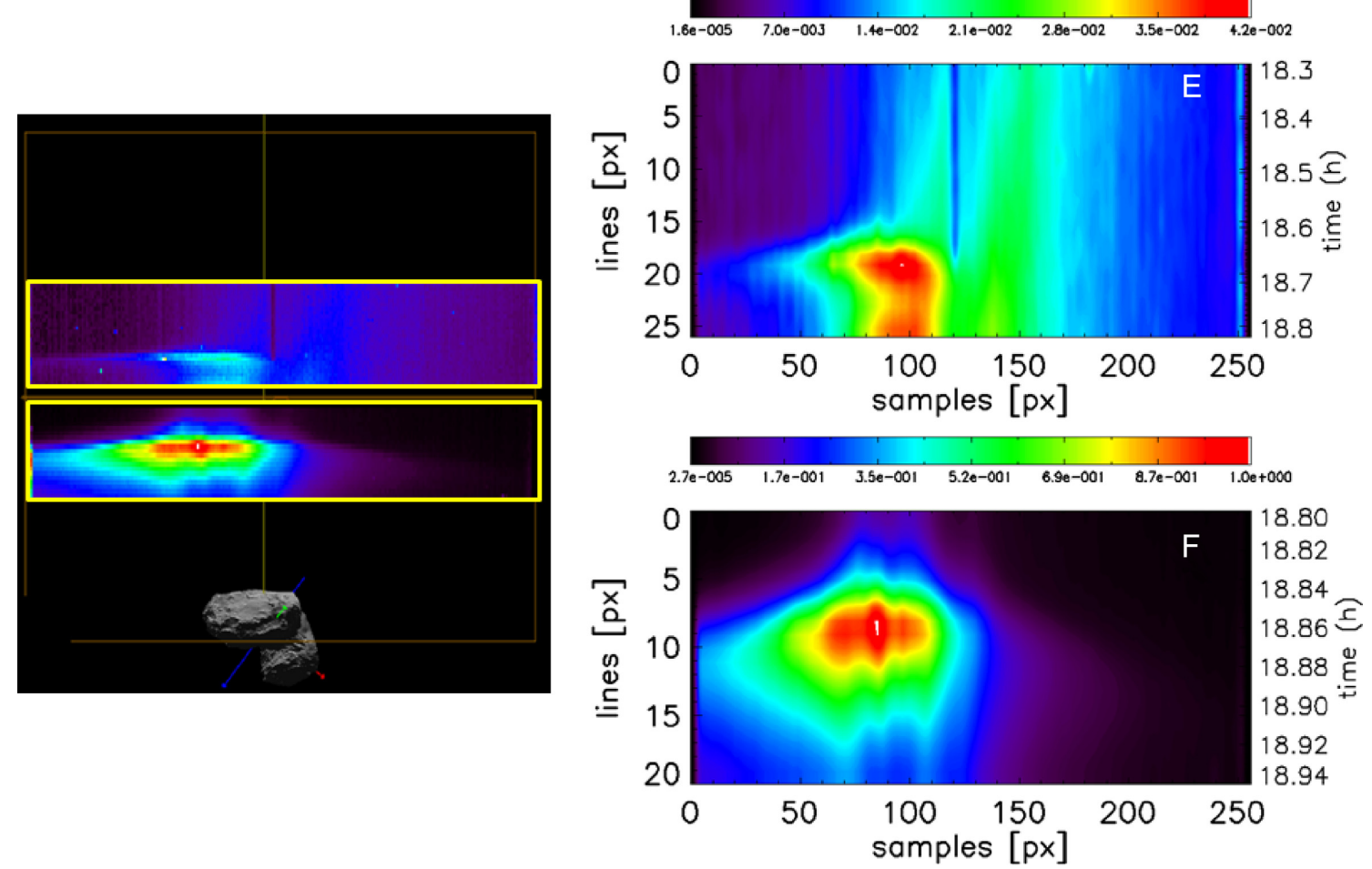

Figure 3. Radiance value at $0.55 \mu \mathrm{m}$ for the images V1_00400875328 (E) and V1_00400877229 (F) acquired on 2015 September 14 (images on the right), showing the nucleus, dust coma, and outburst ejecta (collimated ejecta). The images on the left show the configuration of the nucleus of the comet with respect to the $\mathrm{VM}$ frames (yellow rectangles). The radiance has units of $\mathrm{W} \mathrm{m}^{-2} \mathrm{sr}^{-1} \mu \mathrm{m}^{-1}$.

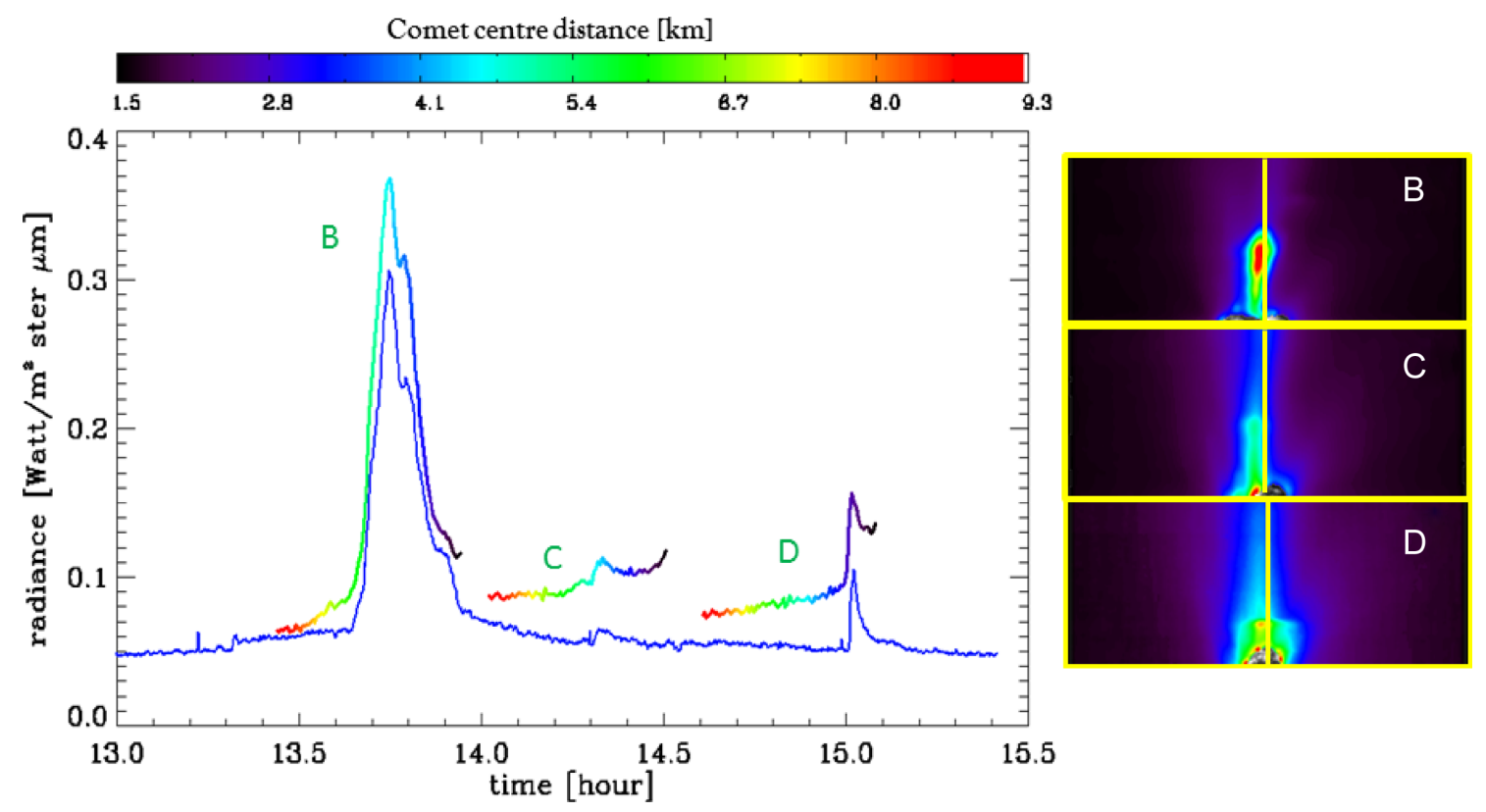

Figure 4. Light curves of the outburst sequence of 67P/CG during 2015 September 13. The blue curve is the VH light curve radiance at $3.85 \mu \mathrm{m}$ (multiplied by 30). The multicolour curves are the VM radiances at $0.55 \mu \mathrm{m}$. The images on the right show the VM image frame for the observations $\mathrm{B}, \mathrm{C}$, and $\mathrm{D}$, and the yellow lines in the middle of the frame (sample 126) show where the VM profiles have been extracted. The different colours used for the VM light curves refer to the distance from the comet centre.

$45 \mathrm{~min}$. Since each line of the image was acquired $20 \mathrm{~s}$ apart from each other, we can analyse, along the vertical dimension of the image, the temporal evolution of the coma components, as shown in Fig. 1, where we display the line number and the corresponding time of acquisition for each line (right-hand side of the images). The figure is a composite image where the comet nucleus, taken as an average in the wavelength range between 0.45 and $0.55 \mu \mathrm{m}$, is superimposed on to the maps of the dust continuum at $0.55 \mu \mathrm{m}$. 

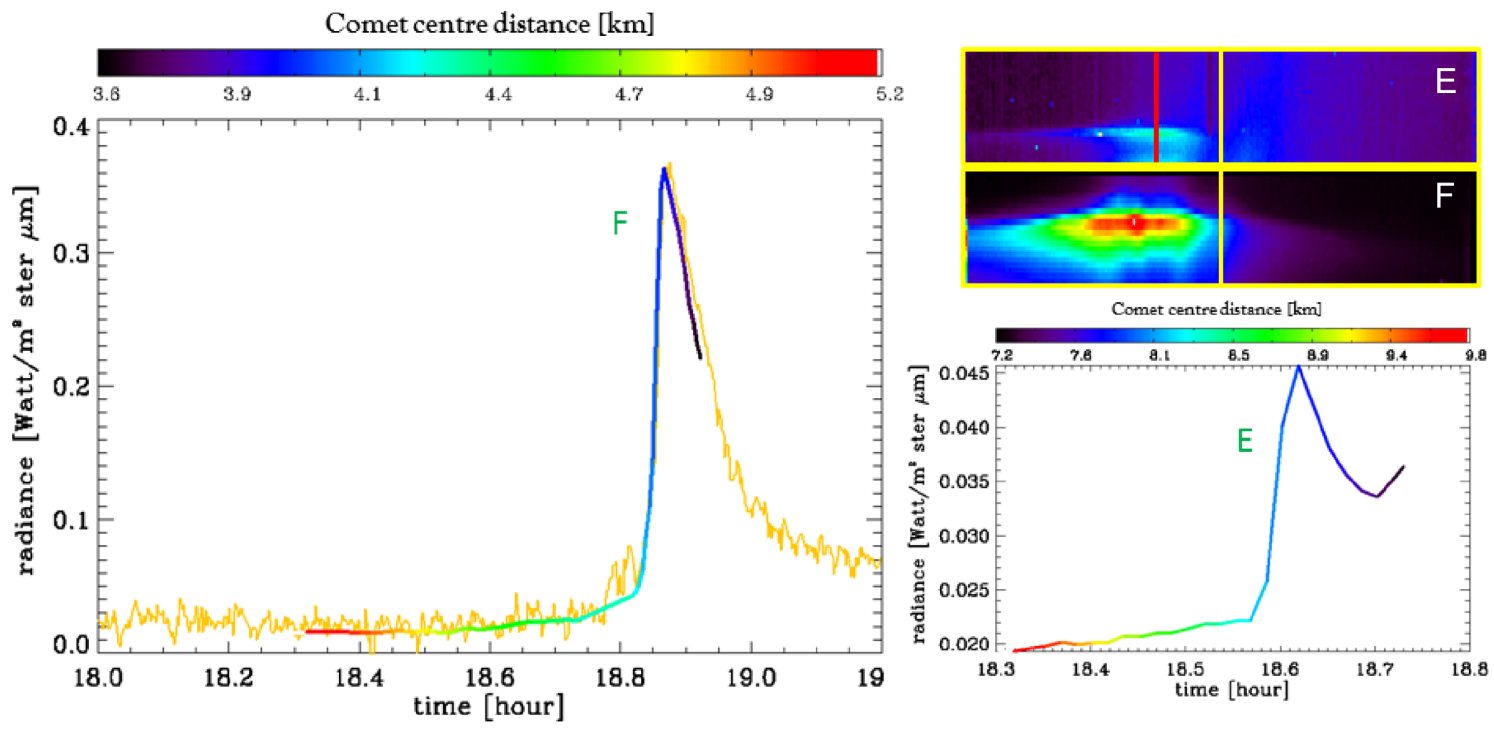

Figure 5. The plot on the left shows the light curve of the outburst F acquired on 2015 September 14. The yellow curve is the VH light curve radiance at 2.42 $\mu \mathrm{m}$ (multiplied by 50). The multicolour curve is the $\mathrm{VM}$ radiance at $0.55 \mu \mathrm{m}$. The images on the right show the $\mathrm{VM}$ image frame for the observations $\mathrm{E}$ and $\mathrm{F}$, and the yellow lines in the middle of the frame (sample 126) show where the VM profiles have been extracted. The plot on the right shows the VM light curve of outburst E extracted along the red line in the frame E. The different colours used for the VM light curves refer to the distance from the comet centre.

Table 2. Outburst properties in the VIS channel.

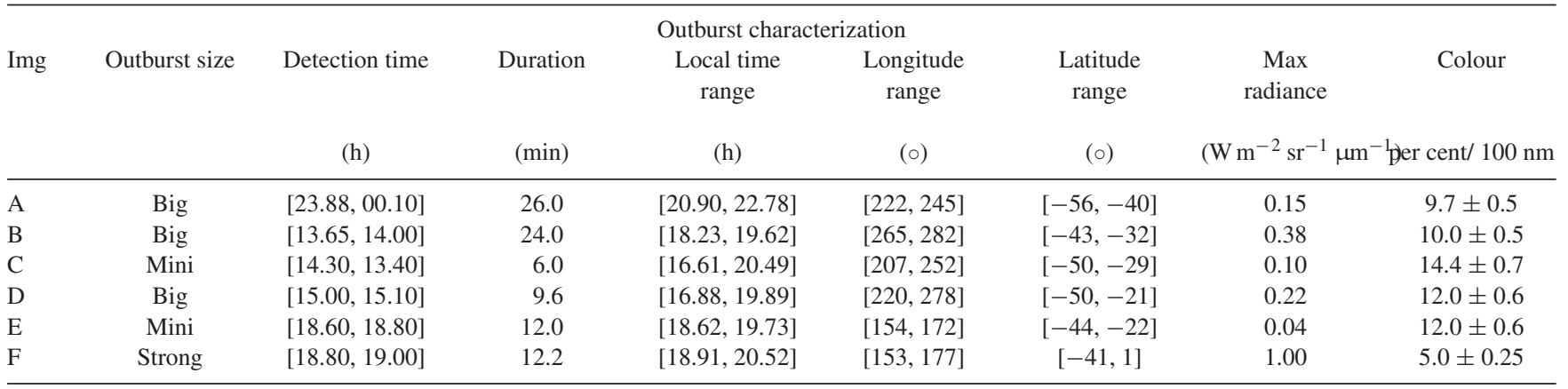

Notes. Img: Assigned letter for each image cube.

Outburst size: Size of outburst defined in terms of radiance level at the maximum (eighth column). 'Big' if the radiance is $>0.1 \mathrm{~W} \mathrm{~m}^{-2} \mathrm{sr}^{-1} \mu \mathrm{m}^{-1}$ and 'mini' if the radiance level is $\leq 0.1 \mathrm{~W} \mathrm{~m}^{-2} \mathrm{sr}^{-1} \mu \mathrm{m}^{-1}$. The outburst (f) is 'Strong' because it is the strongest event observed on 67P/CG (see Section 3).

Time detection: Start and stop detection time for each outburst.

Duration: Lifetime for each outburst.

Local time range: Local time range of outburst source on surface.

Longitude range: Longitude range of outburst source on surface (see Section 4).

Latitude range: Longitude range of outburst source on surface (see Section 4).

Max radiance: Radiance at $0.55 \mu \mathrm{m}$ at the maximum of the outburst emission.

Colour: Colour at the maximum of the outburst radiance (see Section 6).

As in all the VM images reported in this paper, the Sun is shining from the top of the image. In addition to the illumination-driven dust activity, the image shows, on the left, a sharply bound ejecta blob caused by an outburst.

The maximum intensity in the blob is $0.17 \mathrm{~W} \mathrm{~m}^{-2} \mathrm{sr}^{-1} \mu \mathrm{m}^{-1}$, 8.5 times greater than the background coma.

\subsection{The September 13 outburst sequence}

The September 13 outburst sequence is composed of three hyperspectral cubes acquired in $30 \mathrm{~min}$ each at $1 \mathrm{~min}$ intervals (Fig. 2). The dust distribution shows collimated structures whose behaviour is correlated with the illumination conditions, with three consecutive outbursts of dust blobs in the direction of the Sun.
Fig. 2B illustrates the first large event. It appears as a collimated plume in the FOV plane, with a complex pattern showing internal structures, suggestive of the presence of three collimated ejecta originating from a common source on the nucleus and expanding thereafter at a uniform rate. The ejecta of this outburst did not follow a straight line, but a curved trajectory, probably due to the rotation by $7.5^{\circ}$ of the cometary nucleus during the event. This sequence is an example of how the outburst emissions can be clustered in time without strict periodicity between them (Farnham et al. 2007). The maximum intensity of the event is $0.38 \mathrm{~W} \mathrm{~m}^{-2} \mathrm{sr}^{-1} \mu \mathrm{m}^{-1}, 13$ times more intense than the background coma.

In Fig. 2C, we see the onset of another less-intense event, a 'mini' outburst, manifested as a collimated structure with a maximum intensity of $0.10 \mathrm{~W} \mathrm{~m}^{-2} \mathrm{sr}^{-1} \mu \mathrm{m}^{-1}$. 
Finally, a third outburst (Fig. 2D) resulted in a complex event, apparently the result of three or more ejecta sources on different active regions of the surface. The internal structures form a collimated and well-defined structure, with a maximum intensity of $0.22 \mathrm{~W} \mathrm{~m}^{-2} \mathrm{sr}^{-1} \mu \mathrm{m}^{-1}$.

Because of the simultaneous acquisition of the three outbursts by $\mathrm{VH}$, the temporal evolution of the complete sequence can be followed in more detail, by comparing the light curves from the two instruments as shown in Fig. 4.

Throughout the VM scans, VH stared at a fixed point in the coma at $3.6 \mathrm{~km}$ from the nucleus centre (Bockelée-Morvan et al. 2017); thus the VH light curve shown in Fig. 4 (blue curve) represents the time evolution of the radiance at $3.85 \mu \mathrm{m}$. The $\mathrm{VM}$ temporal profiles (multicolour curves) have been extracted in correspondence of the location of the VH FOV along the slit direction of VM (sample 126 in the horizontal direction). The colours used for the VM data points correspond to the colour scale displayed in the figure.

The light curves of the two instruments are remarkably similar and allow us to accurately determine the duration and temporal variation of the intensity for each outburst. The first big outburst is a combination of three consecutive events, at small time intervals, lasting altogether about $24 \mathrm{~min}$, while the two mini outbursts occurred about $40 \mathrm{~min}$ and $80 \mathrm{~min}$ later. Another common behaviour is that all the outbursts are characterized by a sudden increase of the dust radiance, reaching maximum intensity a few minutes later, followed by a smooth decrease. This behaviour is typical of cometary outbursts (Belton et al. 2008; Knollenberg et al. 2016). In both light curves, the big event shows a complex pattern caused by three expanding collimated blobs ejected at 5 min intervals.

Although VM and $\mathrm{VH}$ are fundamentally different in their mode of operation, VH staring at a fixed point in space with the dust contained in the outburst flowing through its FOV and VM scanning spatially and temporally through the outburst, the two light curves display striking similarities. we can draw two important conclusions: First, VM is able to follow the three outbursts during their complete evolution, and secondly, the dust grains observed by $\mathrm{VH}$ at $3.6 \mathrm{~km}$ are same as those VM detected at different distances. This shows how fast the dust is travelling away from the nucleus, as we shall see in more detail in Section 5.

\subsection{The September 14 outburst sequence}

On September 14, VM observed two outbursts, which were captured in two consecutive data cubes acquired 4 min apart. On the left of Fig. 3, we show the configuration of the nucleus of the comet with respect to the location of the FOV of two cubes, at the time of the observation, indicated by the yellow boxes.

Image $\mathrm{E}$ covers the distance from 5 to $4 \mathrm{~km}$ and image $\mathrm{F}$ from 4 to $3.6 \mathrm{~km}$ from the nucleus centre. The first image, shown in better detail on the right-hand side of Fig. 3, shows two jets with a dust maximum emission radiance of $0.024 \mathrm{~W} \mathrm{~m}^{-2} \mathrm{sr}^{-1} \mu \mathrm{m}^{-1}$ and an irregular blob with a maximum emission radiance of $0.042 \mathrm{~W} \mathrm{~m}^{-2}$ $\mathrm{sr}^{-1} \mu \mathrm{m}^{-1}$, two time bigger than the maximum of the radiance jets. The same outburst has been observed by OSIRIS-NAC at $18.78 \mathrm{~h}$ UT. It is the outburst n. 33 in Vincent et al. (2016), a complex event combining two narrow features that arise from the same source region on the surface. The two features are present even in the VM image, the outburst and the jet (Fig. 3E).

Image $\mathrm{F}$ shows a large, intense blob spanning $8 \mathrm{~km}$ in the horizontal direction and $2 \mathrm{~km}$ in the vertical. The maximum dust radiance intensity is $1 \mathrm{~W} \mathrm{~m}^{-2} \mathrm{sr}^{-1} \mu^{-1}$, similar to the typical radiance values measured on illuminated regions of the nucleus surface. All plumes come from the big lobe of the comet and are asymmetrically located relative to it.

The temporal evolution of the coma during the outburst sequence of 2015 September 14 can be followed in more detail by taking a vertical profile through VM data, as shown in Fig. 5. As for the September 13 outburst, the vertical profile has been taken to match the VH FOV (yellow line in the images E and F of Fig. 5), however, in this case only the F outburst has been detected both by $\mathrm{VH}$ and $\mathrm{VM}$, while the $\mathrm{E}$ outburst was only marginally covered by the $\mathrm{VH}$ FOV.

Fig. 5 shows VM and VH light curves of the F outburst that started at $18.80 \mathrm{~h}$ UT and ended at $19.00 \mathrm{~h} \mathrm{UT}$. Unfortunately, the VM curve does not cover the complete evolution of the outburst. The second plot displayed in the right part of Fig. 5 shows the light curve of outburst $\mathrm{E}$ taken along the red line in the image at the maximum radiance level. Outburst $\mathrm{E}$ is a mini outburst starting at $18.57 \mathrm{~h}$ UT. The light curve shows the typical behaviour of outburst evolution, a sudden increase followed by a slower decrease. The subsequent increase in the radiance is probably related to the start of another outburst unfortunately falling outside VM FOV.

\section{OUTBURST LOCATIONS}

In the VM images analysed in this work, the dust ejecta are seen only in one image, and the source location of the outbursts is not visible. Hence, we can only roughly identify the location of the source location. We selected the pixels inside the outburst ejecta and determined the projected latitude and longitude on the surface at the intersection with the vector passing through the centre of the target (tangent point).

Fig. 6 shows the outburst source regions projected on a morphological map of 67P/CG displaying the region boundaries defined by El-Maarry et al. (2015, 2016). All outburst sources are located in the Southern hemisphere, approximately at the latitude range from $-5^{\circ}$ to $-55^{\circ}$.

For outbursts observed on 2015 September 14, the likely source location is where Oklay et al. (2016) detected potential activity sources by analysing OSIRIS images in large parts of the Imhotep region using spectral techniques to detect the potentially active regions based on spectral slopes. Vincent et al. (2016) observed the outburst $\mathrm{E}$ at latitude $-25^{\circ}$ and longitude $198^{\circ}$ that is very close to our detection considering the large uncertainty in the VM mapping.

In the same region, Knollenberg et al. (2016) observed a small nightside outburst on 2015 March 12, and Agarwal et al. (2017) detected an outburst at latitude $-41^{\circ}$ and longitude $158^{\circ}$ on 2015 July 3.

The outbursts observed on 2015 August 10 and September 13 arise from the eastern side of the body around longitude 210-280 and latitude $-20--55^{\circ}$. These areas are characterized by steep scarps, cliffs, and pits with considerable talus deposits (El-Maarry et al. 2016; Pajola et al. 2016). Grün et al. (2016), on 2016 February 19 , observed an outburst in the Atum region at latitude $-28.6^{\circ}$ and longitude $232.3^{\circ}$.

Kramer et al. (2017) present several monthly data sets of the Rosetta's cometary pressure sensor (COPS), which is part of the Rosina payload. They reconstruct the gas density in the coma with an inverse gas model constructed by fitting the COPS measurements to potential gas sources distributed across the entire nucleus surface. After perihelion, they find a correlation of more active gas emitting areas with the locations of dust outbursts observed around perihelion (Vincent et al. 2016). On 2015 September, the outburst locations are close to active gas emitters. 


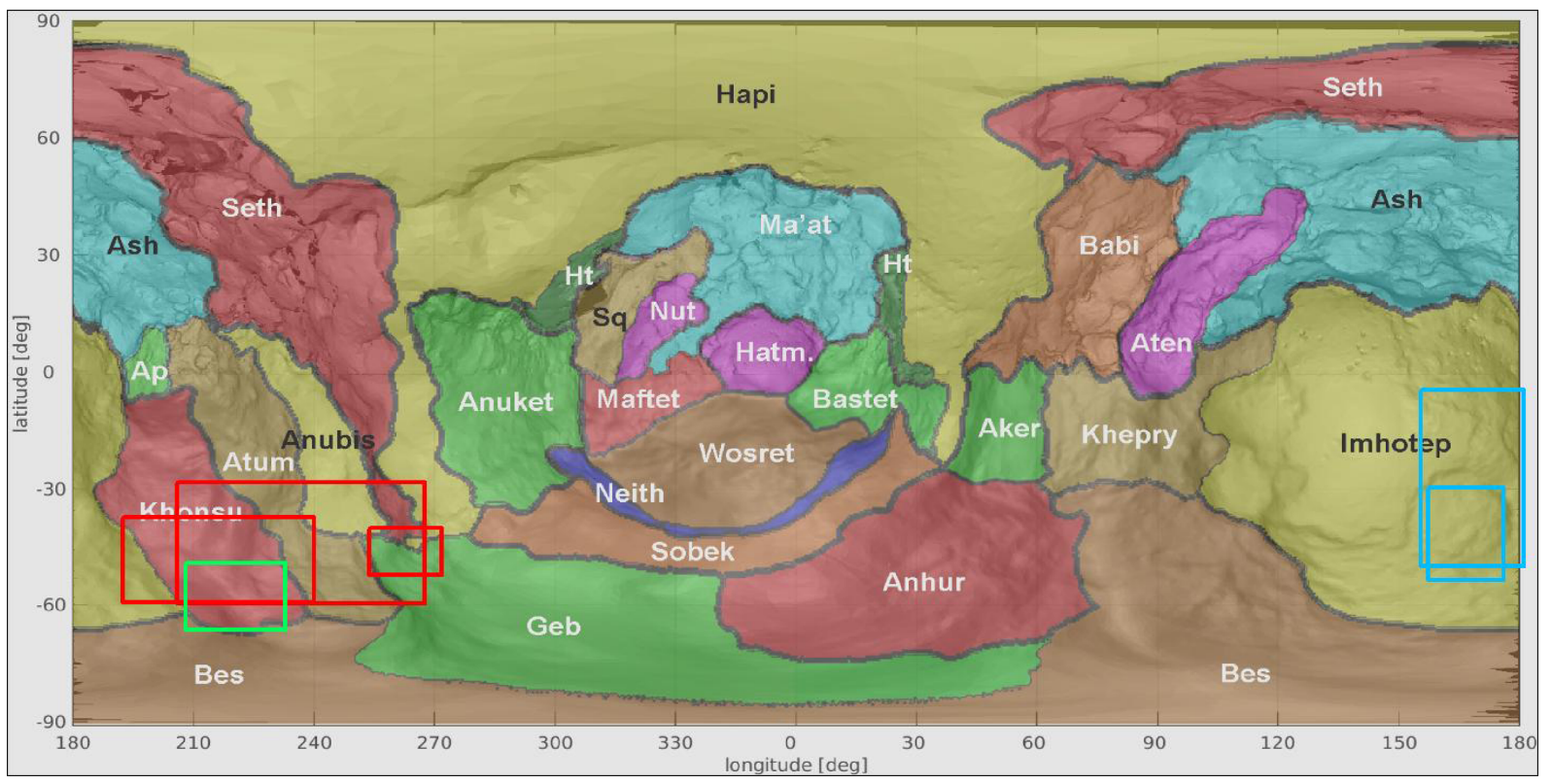

Figure 6. Source regions of the outbursts detected by VM. The red and blue rectangles correspond to the September 13 and 14 sequence, respectively. The green rectangle corresponds to the August 10. The map is centred on the small lobe, the big lobe covers the left-hand and right-hand side of the map, and the contact area between the two lobes covers mainly the top of the map (regions Hapi and Seth). The boundary regions, shown in the map, have been defined by El-Maarry et al. $(2015,2016)$.

The observations by the above-mentioned Rosetta instruments suggest that there are localized areas on the surface that are more prone to outbursts than the rest of the nucleus. In particular, despite the poor statistics, VIRTIS finds that the broad events are likely located in the Imhotep region, while the collimated ones originate from the Atum, Anubis, Khonsu regions. This is an indication that the morphology of outbursts can be correlated with local topography, with flatter areas preferentially giving rise to broad outbursts, while more rugged regions produce more collimated ones.

\section{DYNAMICS OF OUTBURST PARTICLES}

In previous investigations, the expansion speed of the outburst ejecta was often determined using the cadence of the images. Vincent et al. (2016) found that dust plumes, observed by OSIRIS, were ejected with a minimum velocity of $13 \mathrm{~m} \mathrm{~s}^{-1}$.

On 2016 July 3, the Star Tracker cameras on Rosetta detected fast particles travelling at a speed of $(25 \pm 10) \mathrm{m} \mathrm{s}^{-1}$ at the beginning of the event and slower ones at velocity of $(0.41 \pm 0.05) \mathrm{m} \mathrm{s}^{-1} 1 \mathrm{~h}$ after (Agarwal et al. 2017). Similar velocity values were measured for the outburst of 2016 February 19 (Grün et al. 2016).

In this section, we present a simple method to calculate the velocity of outburst ejecta by comparing the VM and VH light curves.

To perform this calculation, we assume, following the discussion at the end of Section 3.2, that VM and $\mathrm{VH}$ are observing the same dust particles. We determined the shifts in time $\left(\Delta_{\mathrm{t}}\right)$ and distance ( $\Delta_{\text {position }}$ ) of the radiance peaks in the VM and VH light curves. The ratio between the two gives us the expansion velocity of the dust particles, projected on the plane of the sky.

\subsection{Outbursts dust speeds derived by VIRTIS data}

\subsubsection{The September 13 outburst dust velocity}

As described in Section 3.2, on September 13 the comet produced a sequence of outbursts. The first one is a big outburst composed by three consecutive emissions of dust ejecta in the direction of the Sun (Fig. 2). All outbursts were detected both by VH and VM.

Fig. 7B contains two plots. The upper one shows the light curves of the big outburst obtained by $\mathrm{VM}$ and $\mathrm{VH}$, at 0.55 and 3.85 $\mu \mathrm{m}$, respectively, and the lower plot shows the distance from the surface of the VM and VH FOVs, where the data were acquired. Since the FOV of VH was at fixed position, $2.6 \mathrm{~km}$ from the comet surface (Fig. 7B, lower plot), the recorded signal represents the real temporal profile of the outburst at the position of the entrance slit. The VM profile has been extracted from the centre of each image sample that corresponds to the position of VH FOV with respect to the VM frame. From the light curves, it is possible to detect the three outbursts: the first one is the strongest, followed by the subsequent weaker, ones in the decay shoulder of the first. Due to the weakness of the third blob in the big outburst in the VM light curve, it is not possible to measure its position or its velocity at the maximum.

Table 3 gives the calculated projected velocity for the first two local maxima of outburst $\mathrm{B}$ and for the $\mathrm{C}$ and $\mathrm{D}$ mini outbursts.

The velocities derived at the maxima of the light curves are $30.2 \pm 1.4$ and $22.2 \pm 2.2 \mathrm{~m} \mathrm{~s}^{-1}$ for the outburst $\mathrm{B}$ and $38.4 \pm 2.1$ and $64.9 \pm 10.6 \mathrm{~m} \mathrm{~s}^{-1}$ for $\mathrm{C}$ and $\mathrm{D}$. The velocity errors corresponding to estimated uncertainties in timing of the order of $1-2 \mathrm{~s}$, while that of the distance is about $30 \mathrm{~m}$.

\subsubsection{The September 14 outburst dust velocity}

On September 14, the comet produced another very large outburst detected both by VH and VM (Fig. 5). We calculate the dust grain expansion velocity inside the outburst applying the same method 

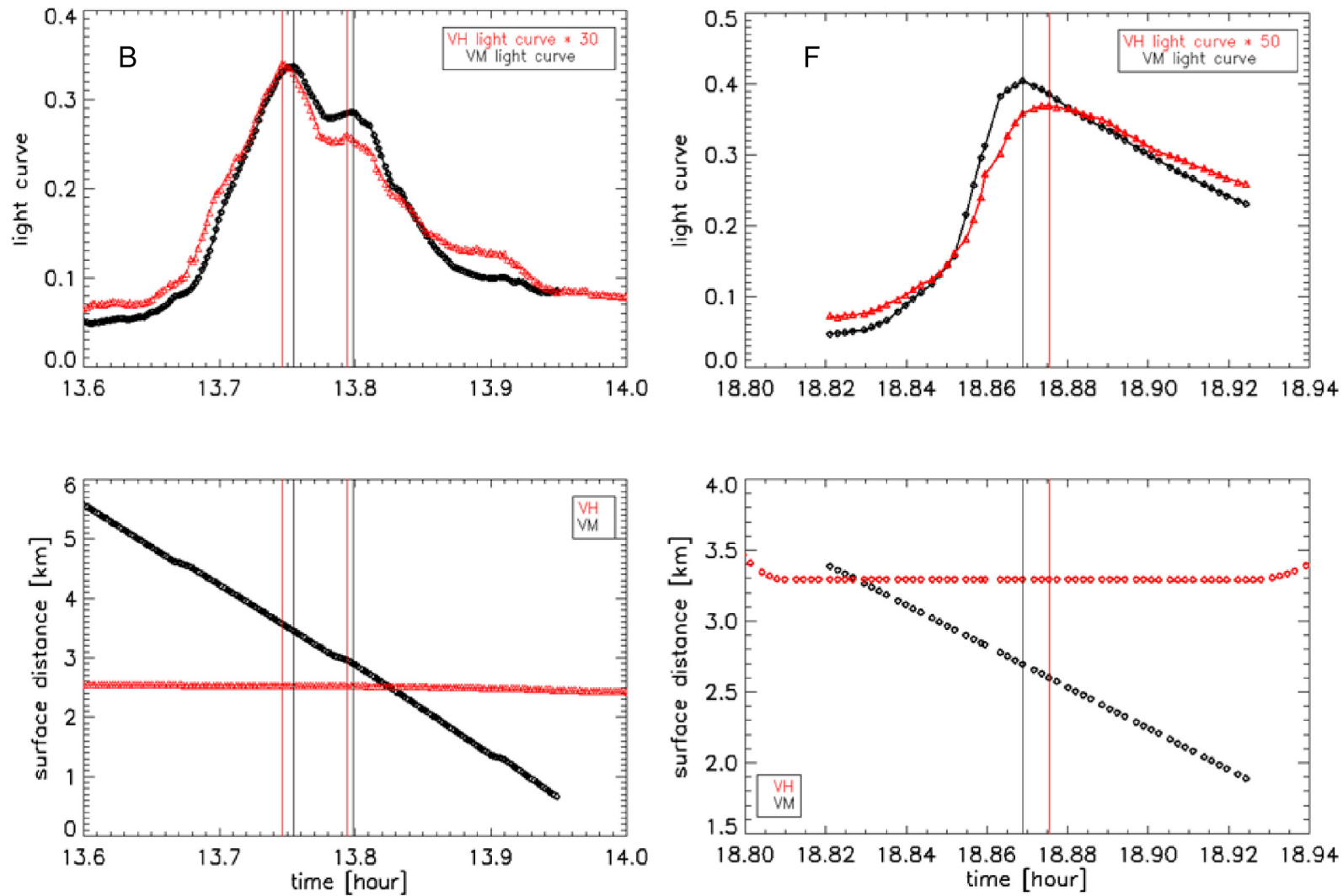

Figure 7. The upper plots show the VM and VH light curves of the big outbursts observed on 2015 September 13 and 14, B and F, respectively. The red curves are the $\mathrm{VH}$ radiance at $3.85 \mu \mathrm{m}$ (multiplied by 30 ) for outburst B and at $2.42 \mu \mathrm{m}$ (multiplied by 50) for outburst F. The black curves are the VM radiance at $0.55 \mu \mathrm{m}$. The lower plots show the distance to the surface of the VH and VM FOV during the observations.

Table 3. September 13 and 14 projected outburst velocity.

\begin{tabular}{lccccc}
\hline Img & $\begin{array}{c}\text { Time }_{\mathrm{H}} \\
(\mathrm{h})\end{array}$ & $\begin{array}{c}\text { Time }_{\mathrm{M}} \\
(\mathrm{h})\end{array}$ & $\begin{array}{c}\Delta_{\mathrm{t}} \\
(\mathrm{s})\end{array}$ & $\begin{array}{c}\Delta_{\text {position }} \\
(\mathrm{m})\end{array}$ & $\begin{array}{c}\text { Velocity } \\
\left(\mathrm{m} \mathrm{s}^{-1}\right)\end{array}$ \\
\hline \multicolumn{7}{c}{ 2015 Sept 13 } \\
B & $13.7462^{\star}$ & 13.7546 & 30.38 & 918 & $30.2 \pm 1.4$ \\
B & $13.7940^{\star}$ & 13.7987 & 16.86 & 374 & $22.2 \pm 2.2$ \\
C & 14.3452 & 14.3386 & 23.72 & 912 & $38.4 \pm 2.1$ \\
D & 15.0258 & \multicolumn{7}{c}{15.0219} & 6.75 & 438 & $64.9 \pm 10.6$ \\
F & 18.8754 & \multicolumn{2}{c}{ 2015 Sept 14} \\
\hline
\end{tabular}

Notes. Img: Assigned letter for each image cube.

Time $_{\mathrm{H}}$ : Recorded time of the maxima in the VH light curves.

Time $_{\mathrm{M}}$ : Recorded time of the maxima in the VM light curves.

$\Delta_{\mathrm{t}}$ : Time difference between the peaks measured by the two instruments.

$\Delta_{\text {position }}$ : Position difference between the peaks measured by the two instruments.

Velocity: Projected velocity for each event.

${ }^{\star}$ Recorded time, position, and velocity of two blobs of outburst B

as for September 13 (Section 5.1.1). Fig. 7F contains the same information as the previous plot, with the $\mathrm{VH}$ profile at $2.42 \mu \mathrm{m}$. In this case, the FOV of $\mathrm{VH}$ is at $3.3 \mathrm{~km}$ from the comet surface (lower plot).

The results are reported in Table 3. As observed on September 13, during this outburst the dust grain velocities are accelerated within a few minutes reaching a velocity of $25.3 \pm 1.65 \mathrm{~m} \mathrm{~s}^{-1}$. The uncertainty in the velocity has been evaluated as in Section 5.1.1.

\subsection{Outburst dust speeds computed by dynamical simulations calibrated with GIADA data}

The aim of this section is to compare the dust speeds derived by the VIRTIS light curves (Section 5.1) with a 3D+t non-spherical dust dynamical model (Fulle et al. 2015; Ivanovski et al. 2017a,b) calibrated with the GIADA measurements (Colangeli et al. 2007; Della Corte et al. 2016). Since VIRTIS observed the coma close to the nucleus, the two main forces acting on the dust particles are the aerodynamic force and gravity.

\subsubsection{Model setup and calibration with GIADA data}

In Table 4, the parameters used in the model are summarized to the simulated 2015 September 13 and 14 outbursts environment. The first set includes the physical properties of the simulated particles. We considered homogeneous, isothermal spheres, oblate and prolate spheroids with aspect ratio $\mathrm{a} / \mathrm{b}=0.2$ and 5.0 , elongation values able to reproduce GIADA data (Ivanovski et al. 2017b). We considered the dust physical properties retrieved by the GIADA measurements of 2015 August outbursts, i.e. the closer outburst to the 13-14 September events (see Table 5). The second set consists of the initial Euler gas flow parameters and the gas production rate constrained by ROSINA data (Fougere et al. 2016). The third set shows the 67P/CG measured physical parameters used for the gravity force computation. 
Table 4. The dust model initial parameters.

\begin{tabular}{|c|c|c|}
\hline Input parameter & Parameter value & Comments \\
\hline \multicolumn{3}{|c|}{ Particle parameters } \\
\hline Particle size (m) & $0.1 \times 10^{-6}-1.0 \times 10^{-5}$ & $\begin{array}{l}\text { Chosen to include the VIRTIS detection size range }(0.25-5 \mu \mathrm{m}) \text { (Drossart et al. 2000; } \\
\text { Coradini et al. 2007) }\end{array}$ \\
\hline Particle density $\left(\mathrm{kg} \mathrm{m}^{-3}\right)$ & 800 & Constrained by the GIADA data (Fulle et al. 2016, 2017) \\
\hline Spheroid aspect ratio, $a / b$ & 0.2 and 5.0 & Compatible with the GIADA measurements (Fulle et al. 2016, 2017) \\
\hline Initial particle orientation (deg) & $45^{\circ}$ & $\begin{array}{l}\text { Compatible with the one used to reproduce particle speeds measured by GIADA } \\
\text { (Ivanovski et al. 2017b) }\end{array}$ \\
\hline Particle temperature, $T_{\mathrm{d}}(\mathrm{K})$ & 250 and 500 & Bockelée-Morvan et al. (2017) \\
\hline \multicolumn{3}{|c|}{ Initial gas field parameters } \\
\hline Speed $\left(\mathrm{m} \mathrm{s}^{-1}\right)$ & 500 & All these parameters are derived by the Euler gas flow solutions \\
\hline Gas composition & $\mathrm{H}_{2} \mathrm{O}+\mathrm{CO}_{2}$ & calibrated with the DCMC 3D+t gas solutions constrained by \\
\hline Total gas production $\left(\mathrm{mol} \mathrm{s}^{-1}\right)$ & $10^{27}$ & ROSINA data (Fougere et al. 2016). \\
\hline Gas stream temperature $(\mathrm{K})$ & 100 & \\
\hline Gas stream density $\left(\mathrm{kg} \mathrm{m}^{-3}\right)$ & $1.0 \times 10^{-6}$ & \\
\hline \multicolumn{3}{|c|}{ 67P/CG parameters } \\
\hline Radius, $R_{\mathrm{N}}(\mathrm{m})$ & $2.0 \times 10^{3}$ & Sierks et al. (2015) \\
\hline Mass, $m_{\mathrm{N}}(\mathrm{kg})$ & $1.0 \times 10^{13}$ & Pätzold et al. (2016) \\
\hline Surface temperature, $T_{\mathrm{N}}(\mathrm{K})$ & 200 & Capaccioni et al. (2015) \\
\hline
\end{tabular}

Table 5. GIADA measurements of particle speeds, masses, and cross-sections performed in 2015 August. The error of the particle speed measurements is less than 6 per cent (Rotundi et al. 2015).

\begin{tabular}{lrccc}
\hline Event day & Event hour & Particle speed $\left(\mathrm{m} \mathrm{s}^{-1}\right)$ & Particle mass $m_{\mathrm{d}}(\mathrm{kg})$ & Particle cross-section $\left(\mu \mathrm{m}^{2}\right)$ \\
\hline $01 / 08 / 15$ & $2: 43 \mathrm{PM}$ & 16.58 & $6.96 \times 10^{-8} \pm 1.99 \times 10^{-8}$ & $3.08 \times 10^{4} \pm 2.51 \times 10^{3}$ \\
$02 / 08 / 15$ & $1: 51 \mathrm{AM}$ & 20.58 & $1.18 \times 10^{-8} \pm 5.44 \times 10^{-9}$ & $4.73 \times 10^{4} \pm 3.01 \times 10^{3}$ \\
$03 / 08 / 15$ & $3: 25 \mathrm{AM}$ & 13.91 & $4.91 \times 10^{-8} \pm 1.12 \times 10^{-8}$ & $1.56 \times 10^{5} \pm 1.55 \times 10^{4}$ \\
$03 / 08 / 15$ & $11: 20 \mathrm{AM}$ & 11.66 & $5.62 \times 10^{-8} \pm 1.37 \times 10^{-8}$ & $3.03 \times 10^{5} \pm 3.23 \times 10^{4}$ \\
$03 / 08 / 15$ & $11: 16 \mathrm{PM}$ & 32.23 & $2.82 \times 10^{-9} \pm 1.19 \times 10^{-9}$ & $6.90 \times 10^{4} \pm 5.50 \times 10^{3}$ \\
\hline
\end{tabular}

\subsubsection{Computed particle speeds for outbursts of September 13 and 14}

The speeds computed for dust sizes from 0.1 to $10 \mu \mathrm{m}$ are ranging from 150 to $10 \mathrm{~m} \mathrm{~s}^{-1}$ (Fig. 8). The velocities derived from the spectral data are ranging from $64.9 \pm 10.6$ to $22.2 \pm 2.2 \mathrm{~m} \mathrm{~s}^{-1}$. The comparison between the computed and derived speeds shows best agreement for prolate and spherical particles with sizes ranging from 0.3 to $5.0 \mu \mathrm{m}$. The spectral analysis (Bockelée-Morvan et al. 2017) and the measured velocities suggest sizes less than 0.3 $\mu \mathrm{m}$. This difference can be explained as follows: The velocities calculated from VM and VH light curves are projected particle velocity, whereas the dust model computes the full particle velocity that can be considered as an upper limit of particle speeds. In addition, we have difference in the velocity due to the particle shape. The speeds of the oblate particles resulted to be higher in comparison with the speeds of the prolate and spherical ones of the same size and bulk density. The smaller is the oblate spheroid the higher is the deviation from the speeds derived by VIRTIS, reaching more than 100 per cent. The difference due to the particle temperature is less than 10 per cent and is present for all the considered shapes.

\section{COLOUR}

VIRTIS IR data have shown that outbursts are associated with possible compositional and particle size changes (Bockelée-Morvan et al. 2017). In the VIS range, these changes might be evidenced by spatial and temporal evolution of the colour.
The parameter we used is the so-called normalized reflectivity gradient, or reddening, measured in per cent/100 nm (Jewitt \& Meech 1986) calculated using the values of the reflectance at two wavelengths $\left(R_{\lambda_{1}}, R_{\lambda_{2}}\right)$. The dust reflectance, $R$, is a dimensionless quantity calculated by dividing the measured scattered light intensity, $I$, by the solar incident flux at the comet. The wavelengthdependent solar flux is taken from Kurucz (1994). The mean reflectance gradient per $100 \mathrm{~nm}, r$, for a particular wavelength interval then becomes

$r=\frac{R_{\lambda_{2}}-R_{\lambda_{1}}}{\lambda_{2}-\lambda_{1}} * \frac{200}{R_{\lambda_{2}}+R_{\lambda_{1}}}$.

The reflectivity at the two selected wavelengths $\left(\lambda_{1}=550 \mathrm{~nm}\right.$, $\lambda_{2}=750 \mathrm{~nm}$ ) is an average over a narrow band pass of $10 \mathrm{~nm}$ for optimal signal-to-noise ratio (SNR).

We obtained 2D colour maps by measuring the colour for each pixel in the image, see Figs 9, 10, and 12. The colour maps were generated by stretching the contour scale; however, the colour scale in the maps is adapted to each image to increase the contrast.

The colour uncertainties are evaluated by propagating the formal errors and is inversely proportional to the SNR. Inside the outburst ejecta, where the SNR is higher, the colour uncertainty is about 5 per cent, while outside the uncertainty is higher, at 20 per cent. For this reason, the colour fluctuations outside the outburst, region delimited by the contour lines, are not real.

\subsection{Dust colour in August 10 outburst ejecta}

Fig. 9 shows the colour image corresponding to 2015 August 10 . We see that a bluer feature characterizes the front of the outburst (left 

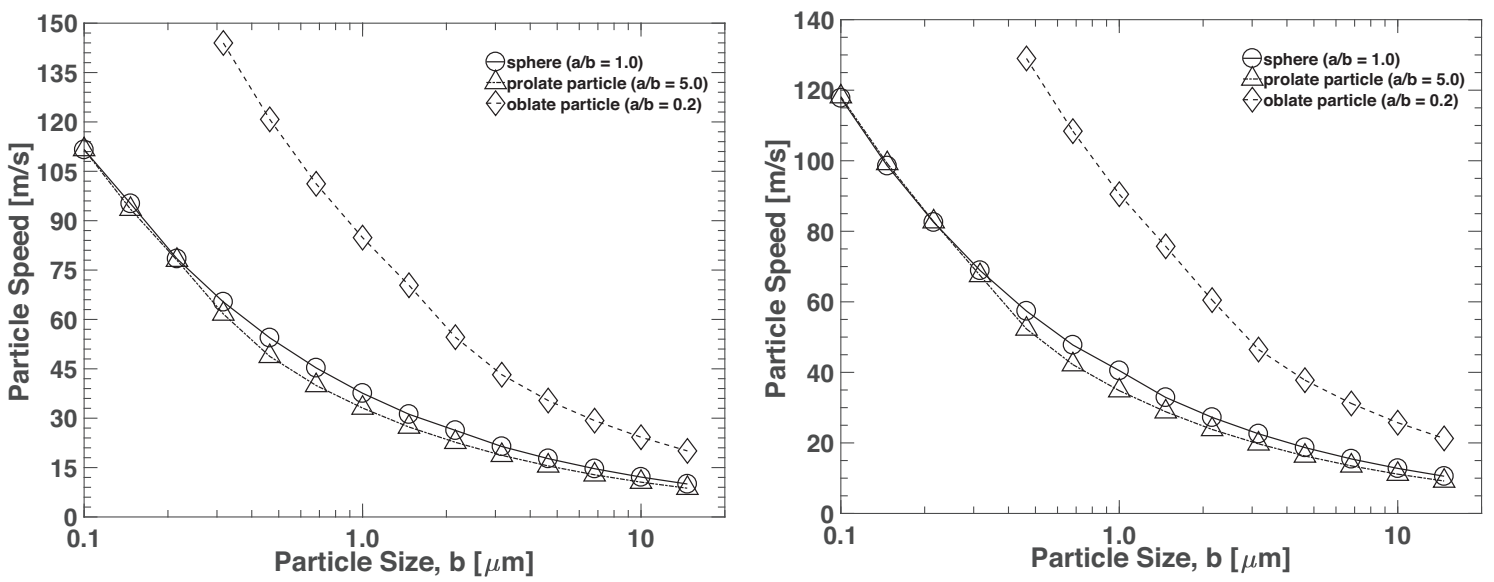

Figure 8. Speeds of particles with initial temperature $250 \mathrm{~K}$ (left) and $500 \mathrm{~K}$ (right) as function of the particle size $b$. The physical properties of the particles are described in Table 4. Three particle shapes are considered, i.e. spheres and spheroids with aspect ratio 0.2 and 5.0 and of bulk density $800 \mathrm{~kg} \mathrm{~m}^{-3}$.
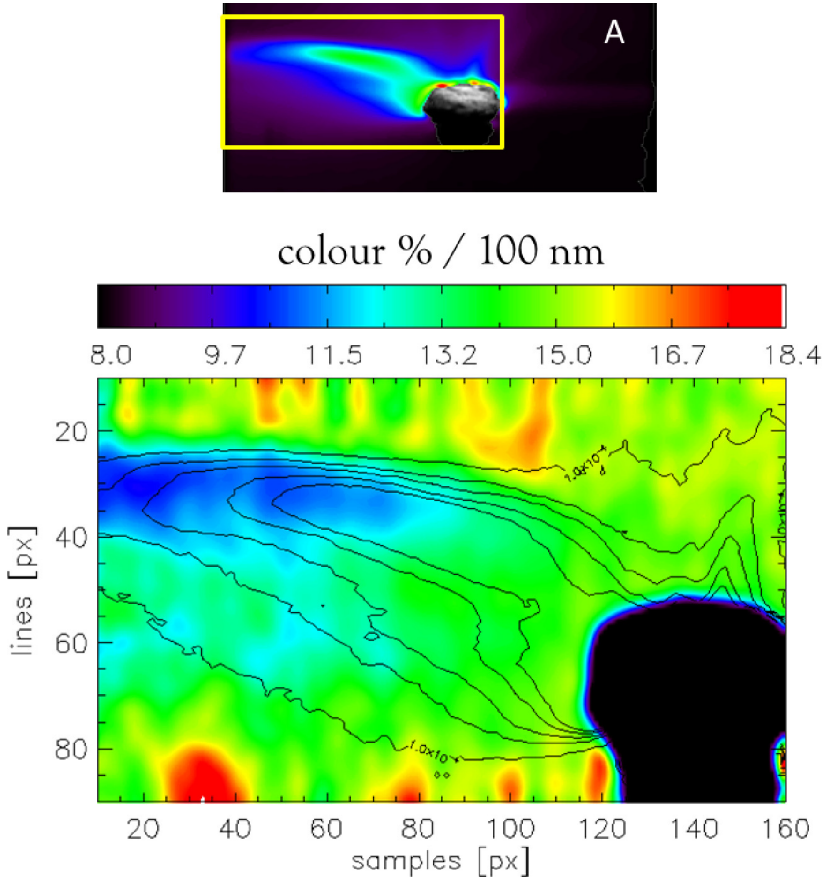

Figure 9. Dust continuum image of the August 10 outburst at $0.55 \mu \mathrm{m}$ (upper plot), and the spatial distribution of the colour (lower plot) calculated in the yellow square of the dust image. The black contours are the radiance levels at $0.55 \mu \mathrm{m}$. The outburst ejecta display colours bluer than the background coma.

part of the ejecta), with colour value of $10 \pm 0.5$ per cent $/ 100 \mathrm{~nm}$, whereas elsewhere the colour is $13-14 \pm 2.5$ per cent $/ 100 \mathrm{~nm}$.

A colour gradient is seen inside the outburst ejecta that arises from a difference in the relative contributions of scattered light from different particle sizes or grain composition. Bluer colours are expected in the presence of an excess of small particles or icy grains (Bockelée-Morvan et al. 2017).

A jet coming from the lit part of the comet surface is observed in the middle right of the image. Its colour, 14-15.5 \pm 0.7 per cent/100 nm, is typical of values measured for the quiescent coma, indicating that the properties of the dust, most probably the particle size distribution, in the jet and in the outburst are different.

\subsection{Dust colour in September 13 outburst ejecta}

Fig. 10 presents the reddening images corresponding to the outburst of 2015 September 13. As described in Section 3.2, the September 13 sequence is composed by three consecutive outbursts with emission of dust blobs in the direction of the Sun.

The colour map of the first big outburst presents a clear colour gradient (Fig. $10 \mathrm{~B}$ ) with the bluer regions being directly associated with the maximum radiance of the internal features with colour values ranging between $10 \pm 0.5$ and $12 \pm 0.6$ per cent $/ 100 \mathrm{~nm}$.

Fig. 11 shows a reflectance spectrum of the big event of September 13 taken at $13.8 \mathrm{~h}$. The reflectance spectrum has been obtained by combining data from the VIS channel of VM and IR channel of $\mathrm{VH}$ in the range from 0.3 to $5.0 \mu \mathrm{m}$. It can be seen that in the VIS and IR regions two reflectance gradients fit the data quite well from about $0.3-0.8 \mu \mathrm{m}$ to $1-2.5 \mu \mathrm{m}$. The reflectance spectrum has a slope with value of $10 \pm 0.5$ per cent $/ 100 \mathrm{~nm}$ from 0.55 to 0.75 $\mu \mathrm{m}$ (Fig. 10) and a slope of $1 \pm 0.3$ per cent/ $100 \mathrm{~nm}$ from 2.2 to $2.5 \mu \mathrm{m}$, reaching a blue colour of $-2.1 \pm 0.1$ per cent/ $100 \mathrm{~nm}$ with a reference pre-outburst removed.

The IR spectra of the outburst ejecta are characterized by a colour temperature of $528 \pm 10 \mathrm{~K}$, retrieved by fitting the thermal part, and a bolometric albedo of $0.67 \pm 0.01$ (Bockelée-Morvan et al. 2017). The combination between the IR and VIS dust colour gradient and the large increase of the colour temperature from $300 \pm 10 \mathrm{~K}$, in the quiescent coma, to $528 \pm 10 \mathrm{~K}$, in the outburst material, reveals the presence of very small grains $(\sim 0.1 \mu \mathrm{m})$ in the outburst ejecta. In addition, the increase of bolometric albedo from $0.12 \pm 0.01$ to $0.67 \pm 0.01$ indicates the presence of bright grains in the ejecta, which could be composed either of silicate, implying the thermal degradation of the carbonaceous material, or ice (Bockelée-Morvan et al. 2017).

However, in the IR spectra, the water ice absorption band at 3 $\mu \mathrm{m}$ is not detected (Bockelée-Morvan et al. 2017).

The far-ultraviolet spectrograph, Alice, onboard Rosetta observed the same outburst in the range between 70 and $205 \mathrm{~nm}$. The spectra show dust in the plume that is brighter than the sunlit nucleus, and that displays a strong absorption feature around $170 \mathrm{~nm}$. This feature is characteristic of water ice (Steffl et al. 2016), suggesting that the observed 'dust' is likely to consist primarily of icy grains.

The absence of a corresponding feature at $3 \mu \mathrm{m}$ would be consistent with the presence of very small icy dust particles with radii less than $100 \mathrm{~nm}$ (Bockelée-Morvan et al. 2017). The UV observa- 

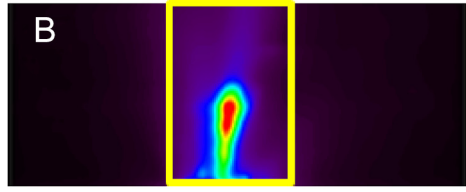

colour $\% / 100 \mathrm{~nm}$
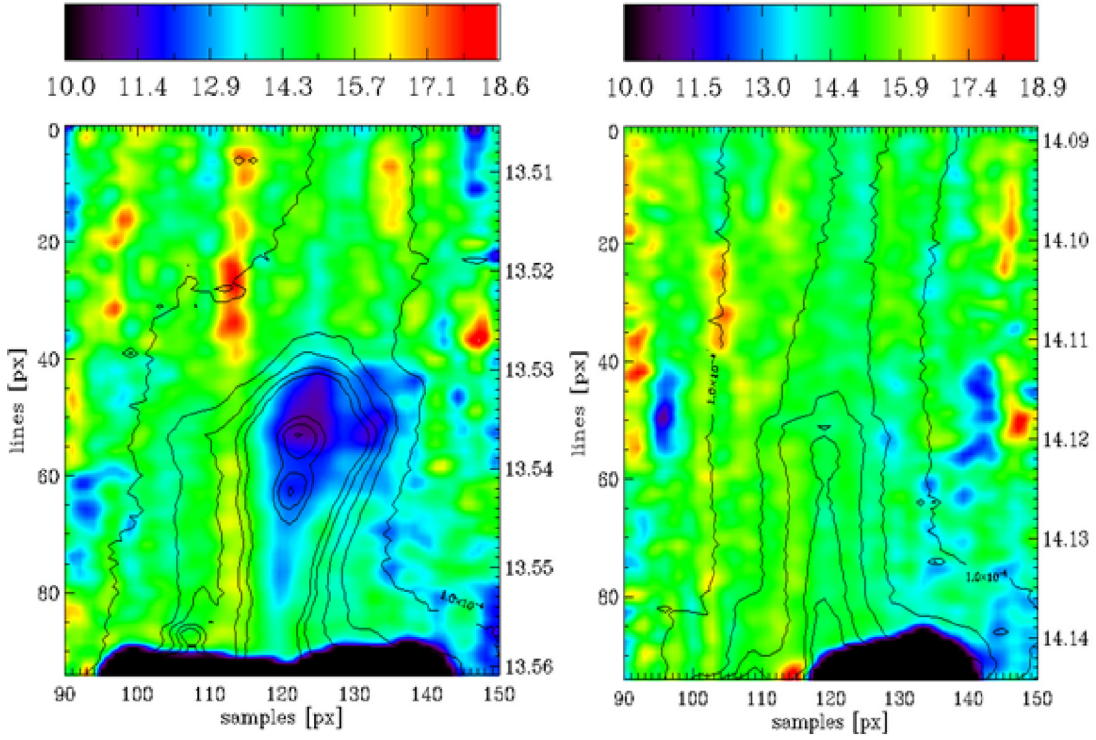

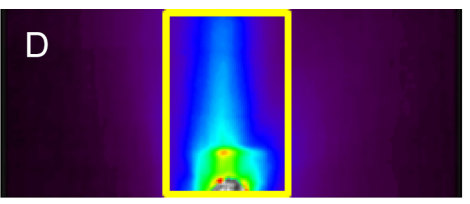

colour $\% / 100 \mathrm{~nm}$

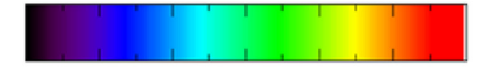

$\begin{array}{llllllll}10.0 & 11.6 & 13.1 & 14.7 & 16.2 & 17.8 & 19.3\end{array}$

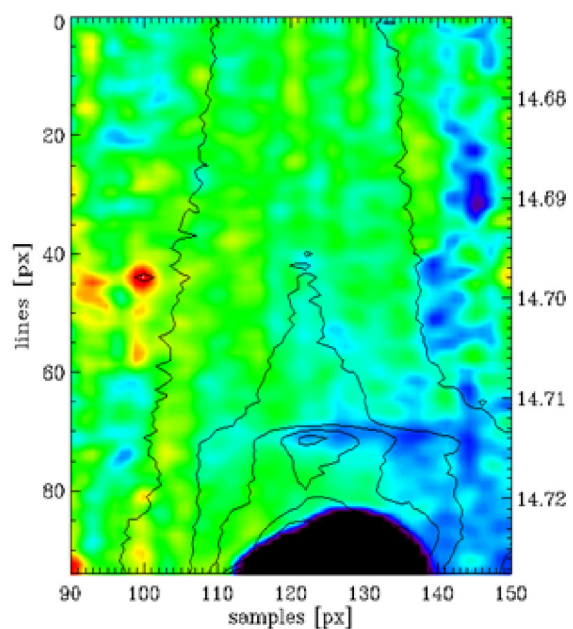

Figure 10. Images of the outburst of September 13 sequence in the VIS at $0.55 \mu \mathrm{m}$ (upper plots), and the VIS spatial distribution of the colour (lower plots) calculated in the yellow square of the dust image. The outburst ejecta B and D display colours bluer than the background coma. However, such variations are not seen in outburst $\mathrm{C}$.

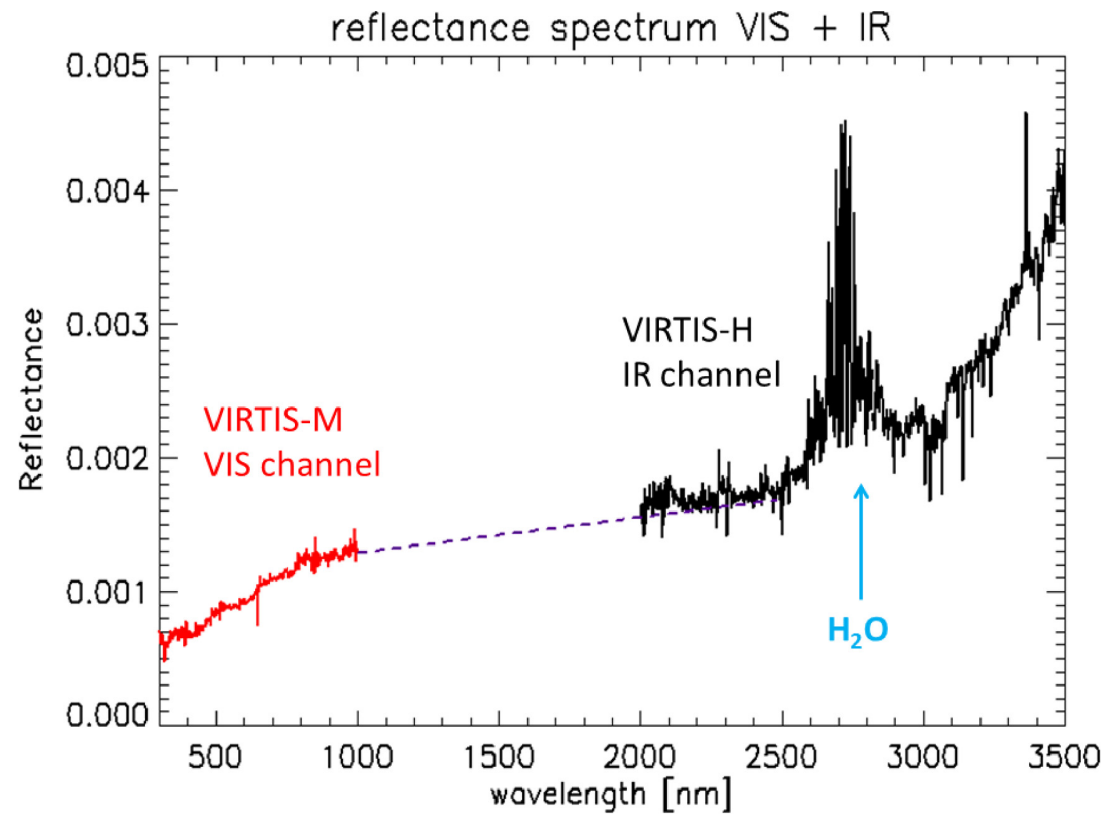

Figure 11. Reflectance spectrum of September 13 outburst ejecta obtained by combining VM and VH data. The spectra were taken at $13.8 \mathrm{~h}$ UT, when VH and VM were observing at the same distance. It can be seen that, in the VIS and IR channels, two colour indices fit the data quite well from about 300 to $800 \mathrm{~nm}$ and from 1000 to $2500 \mathrm{~nm}$, respectively. 

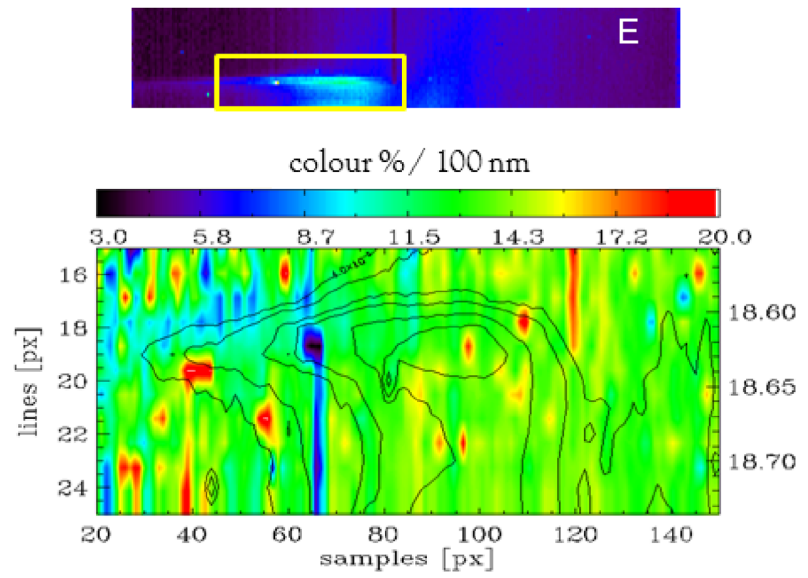
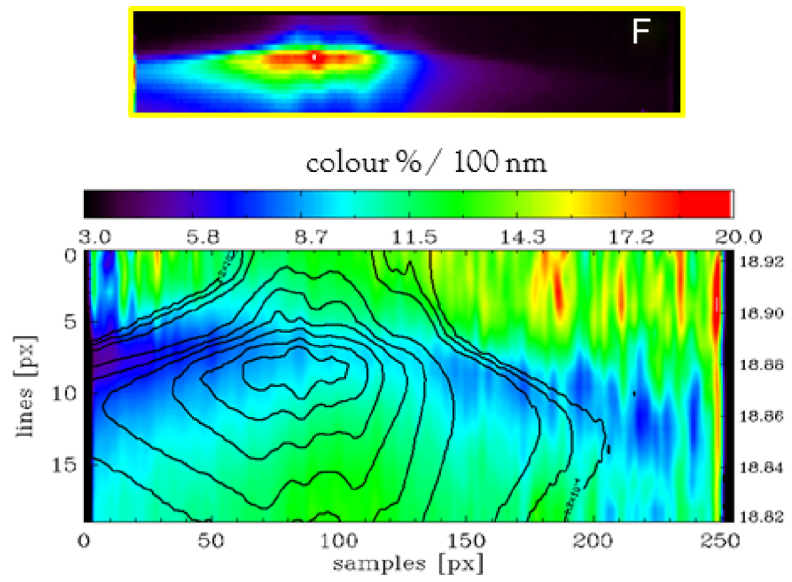

Figure 12. Images of the outburst of September 14 in the VIS at $0.55 \mu \mathrm{m}$ (upper plots) and the VIS spatial distribution of the colour (lower plots) calculated in the yellow square of the dust image. The outburst F displays colours bluer than the background coma, but such variations are not seen in outburst E.

tions are more sensitive to the presence of small particles than the infrared.

For outburst $\mathrm{C}$, we found no clear evidence of different reddening values in the dust continuum with respect to the surrounding coma. The reason is probably that this is a faint outburst and the signal from the background coma dominates, so the colour of the outburst is not measurable.

The colour map of outburst D shows a VIS colour gradient at the maximum of the radiance of the outburst ejecta with a value of $12.0 \pm 0.6$ per cent $/ 100 \mathrm{~nm}$. The bluer colour is evident also in the right-hand part of the colour map, indicating that this outburst is a complex event formed by more than one source at surface (Fig. 2 D). The IR continuum emission of the outburst material $\mathrm{D}$ is characterized by high colour temperatures of $549 \pm 12 \mathrm{~K}$, a bolometric albedo of $0.62 \pm 0.04$, and IR colours $2.2 \pm 0.4$ per cent $/ 100 \mathrm{~nm}$ and a blue value of $-2.2 \pm 0.7$ per cent $/ 100 \mathrm{~nm}$ with a reference pre-outburst removed. Again in this case, the high colour temperatures and blue colour imply the presence of very small grains (Bockelée-Morvan et al. 2017).

\subsection{Dust colour in September 14 outburst ejecta}

The colour images corresponding to the outburst of 2015 September 14 are shown in Fig. 12.

For outburst E, we found no clear evidence of different reddening values in the ejecta cloud with respect to the surrounding coma (Fig. 3). As for outburst $\mathrm{C}$ in the previous figure, the outburst is faint and the dominant spectral variation is either from the jet, on the right of the outburst (Fig. 3) or from the background coma, and the outburst colour properties are not measurable.

For outburst F, the colour in the front of the outburst is bluer than the colour below and above it, especially in the left-hand part of the image. The colour close to the maximum intensity is around $7 \pm 0.35$ per cent $/ 100 \mathrm{~nm}$, while the colour of the decay part is $(10.0-14.0) \pm(0.5-0.7)$ per cent/100 nm. The same colour behaviour was observed by VH in the IR channel. Bockelée-Morvan et al. (2017) found that the evolution of the colour reaches the value of $-2.6 \pm 1.0$ per cent $/ 100 \mathrm{~nm}$, at the peak of intensity, considering the total spectrum and the extreme value of $-9.1 \pm 1.4$ per cent/100 nm removing the pre-outburst spectrum. The IR continuum emission is also characterized by high colour temperatures of $621 \pm 12 \mathrm{~K}$, and a bolometric albedo of $0.56 \pm 0.01$. These prop- erties are interpreted as due to a change of size distribution, with the front part dominated by smaller and brighter particles more abundant because they are faster if accelerated by gas drag or other processes. It is also interesting to note that the distribution of the small grains follows the same broad pattern of the outburst itself.

\section{FILLING FACTOR}

The amount of light scattered by the dust for each pixel is quantified by $A f$, where $f$ is the filling factor or the fraction of a pixel projected area occupied by dust and $A$ represents $Q_{\text {sca }} * p(g), Q_{\text {sca }}$ is the scattering efficiency, $p(g)$ is the phase function normalized over solid angle to $4 \pi$ and $g$ is the phase angle.

Using the formalism applied in Fink \& Rubin (2012), Fink \& Rinaldi (2015) and Rinaldi et al. (2016), the expression to compute the filling factor is

$f=\frac{4 \pi I_{\mathrm{obs}}(a, \lambda)}{F_{\mathrm{i}}(\lambda) A}$,

where $\mathrm{I}_{\mathrm{obs}}\left(\mathrm{W} \mathrm{m}^{-2} \mathrm{sr}^{-1} \mu \mathrm{m}^{-1}\right)$ is the observed scattered light intensity by the instrument, $a$ is the dust particle size, and $F_{i}(\lambda)$ is the incident solar flux.

An approximate method to compute the filling factors is to assume an albedo for the quiescent dust coma and a higher albedo for the outburst dust particles (Agarwal et al. 2017; Bockelée-Morvan et al. 2017). For the quiescent dust coma albedo, we use the quantity $w p(g)$ calculated by Ciarniello et al. (2015) where $w=Q_{\text {sca }} / Q_{\text {ext }}$ is the single scattering albedo at $0.55 \mu \mathrm{m}$. The extinction efficiency $Q_{\text {ext }}$ is equal to 1 , and so $w$ is just the scattering efficiency $Q_{\text {sca. }}$.

In Table 6, we report the values for the quiescent coma and outburst material used for the filling factor computations for different phase angles. For outburst $\mathrm{C}$ and $\mathrm{E}$, we use the values for quiescent coma because the colour maps do not show brighter dust particles.

The filling factor values were obtained at the maximum of the outburst radiance where the values of the filling factor are 2.5 and 5 per cent for the big events and 1.3 per cent for the mini one (outburst D). The high radiance values are not due to a concentration of the larger dust particles but essentially to an abundance of small and bright dust particles with a high geometric albedo, as shown in Bockelée-Morvan et al. (2017). 
Table 6. Filling Factor.

\begin{tabular}{lcccc}
\hline Img & $\begin{array}{c}\text { Phase angle } \\
(\circ)\end{array}$ & $A_{\mathrm{n}}$ & $A_{\text {outburst }}$ & $\begin{array}{c}f \\
\text { (per cent) }\end{array}$ \\
\hline A & 89.00 & 0.04 & 0.24 & 2.5 per cent \\
B & 108.00 & 0.02 & 0.12 & 3.0 per cent \\
C & 109.00 & 0.02 & - & 6.0 per cent \\
D & 109.00 & 0.02 & 0.12 & 1.3 per cent \\
E & 98.84 & 0.04 & - & 1.4 per cent \\
F & 98.71 & 0.04 & 0.24 & 5.0 per cent \\
\hline
\end{tabular}

Notes. Img: Assigned letter for each image cube.

Phase angle: Phase angle for each observation.

$A_{\mathrm{n}}$ : A for nucleus and quiescent coma for each observation taken from Ciarniello et al. (2015).

$A_{\text {outburst }}$ : A for outburst material.

$f$ : Computed outburst filling factor at the maximum of the radiance.

\section{TOTAL DUST MASS EJECTED}

The determination of the total dust mass ejected from an outburst depends on a lot of assumptions, and so the final total mass has a fairly large uncertainty. Uncertainties include the estimate of the number of particles in the plume using scattering theory, and thus the single scattering albedo used, the phase effect, their particle size, their density, the minimum and maximum particle sizes considered in the outburst, the assumption that the particle size distribution and the production rate are same during the duration of the outburst.

For our calculations, we convert the filling factor to a total dust mass ejected, assuming the differential the size distribution of the dust grains $(n(a) \mathrm{d} a)$ and the particles bulk density $(\rho)$.

$f=\frac{N \sigma_{\text {geom }}}{d^{2}}$,

where $N$ is the number of particles inside the pixel area $d^{2}$ and $\sigma_{\text {geom }}$ is the geometrical particle cross-section. The mass of material responsible for the VIS dust emission inside the pixel area $d^{2}$ is computed with the same equations reported by Jewitt \& Luu (1990) and Boissier et al. (2012):

$M_{\text {pixel }}=\frac{4}{3} \pi \rho f d^{2} \frac{\int_{\mathrm{a}_{\min }}^{\mathrm{a}_{\max }} a^{3} n(\mathrm{a}) \mathrm{d} a}{\int_{\mathrm{a}_{\min }}^{\mathrm{a}_{\max }} \pi a^{2} n(a) \mathrm{d} a}$,

where $a_{\min }$ and $a_{\max }$ are the minimum and maximum grain radii. The minimum and maximum size considered in this work are $\mathrm{a}_{\min }$ $=0.1 \mu \mathrm{m}$ and $\mathrm{a}_{\max }=1000 \mu \mathrm{m}$, taking into account the presence of a wide range of particle sizes.

Considering the formulas above, the total observed dust mass ejected during the life-time of the outburst is the integral over a large area containing the outburst. By integrating the mass for each pixel $\left(M_{\text {pixel }}\right)$ over the area where signal from the outburst ejecta is present, the observed total dust mass emitted during the outburst can be determined. In the calculations, we use a constant bulk density of $795 \mathrm{~kg} \mathrm{~m}^{-3}$ for all particles (Fulle et al. 2016).

For outbursts A, B, D, and E, we assume a higher albedo in the outburst regions where the colour maps show brighter particles and a nucleus albedo for the outburst particles outside the bluer regions. For outbursts $\mathrm{C}$ and $\mathrm{E}$, we use only the values for nucleus albedo because the colour maps do not show brighter dust particles (see Figs 9, 10, 12, and Table 6).

For August 10 and September 13 events, we integrated over a rectangle extending from the closest nucleus boundary chosen to avoid capturing any signal from the nucleus to the final edge of the outburst.

The total mass computations are reported in Table 7. The com- putations include a range for the power-law index from -4.5 to -2 because the size distribution within the outburst is unknown.

With this index between -4.5 and -3.5 , the major contribution to the ejected mass is by small particles with radii between 0.1 and $10.0 \mu \mathrm{m}$, while the larger particles contribute most of the mass when the power-law index falls between -3 and -2 .

In the case of the September 14, outburst F, Fig. 3 shows the large blob on the left-hand side indicating that both in the horizontal direction and in the vertical direction (see Fig. 5) the FOV does not cover the complete evolution of the outburst. A fraction of the event is outside the FOV. An approximate estimation gives us about 30 per cent of the mass outside the FOV.

Other estimations of the dust mass ejected from 67P/CG outbursts have been derived from OSIRIS images obtained less than few minutes after outburst onset (Grün et al. 2016; Knollenberg et al. 2016; Vincent et al. 2016; Agarwal et al. 2017; Gicquel et al. 2017). Even if they use different methods and assumptions, all estimations derived outburst mass release of several tons. The main difference from our calculation is that they make the assumption that the outburst geometric albedo is similar to that of the nucleus and quiescent coma. However, we have demonstrated that the high values of radiance at the maximum of the event is mainly due to small and bright dust particles with a higher albedo (Bockelée-Morvan et al. 2017).

Using a size distribution index of -2.5 and a constant bulk density of $1000 \mathrm{~kg} \mathrm{~m}^{-3}$ for all particles, Knollenberg et al. (2016) found a total dust mass of about $300 \mathrm{~kg}$ was produced during the first $3 \mathrm{~min}$ after the outburst became visible.

Using a similar method but assuming a dust size distribution with a power law of -2.6 , Vincent et al. (2016) estimated an ejected flux of 60 for particles in the range $1-10 \mu \mathrm{m}$ or $260 \mathrm{~kg} \mathrm{~s}^{-1}$ for $1-50 \mu \mathrm{m}$. Then, the maximum mass of dust ejected by the event on July 29 was the range from 20 to 80 tons.

Grün et al. (2016) computed the total mass of ejected dust for February 19 outburst and estimated an ejected dust mass of about 1.6 tons, assuming particles size $>100 \mu \mathrm{m}$ detected by GIADA without considering mass contribution by smaller and much bigger particles.

Gicquel et al. (2017) analysed the images from the OSIRISNAC camera to study a bright outburst observed in the Southern hemisphere on 2015 July 29. They found a total mass of ejected dust particles correspond to 20 tons.

In Agarwal et al. (2017), the total mass of ejected dust is estimated to lie between 6.5 and 118 tons, for an outburst life-time of 14-68 min and assuming a constant production rate. The mass range results from the uncertainty of the average density values, ranging from the COSIMA value of $250 \mathrm{~kg} \mathrm{~m}^{-3}$ to the GIADA estimate of $795 \mathrm{~kg}$ $\mathrm{m}^{-3}$ (Fulle et al. 2016).

\section{CONCLUSIONS}

The Rosetta mission to comet 67P/CG has given us the opportunity to observe the coma within few $\mathrm{km}$ from the surface and to study the dynamical and physical properties of the dust in the various outbursts that took place during the active phase of the mission. In this paper, VM observations undertaken on 2015 August 10, September 13 and 14 have been analysed, when the comet was surrounding the perihelion passage at heliocentric distances from 1.2 (inbound) to $1.3 \mathrm{au}$ (outbound). We analysed the temporal brightness profile of the dust plumes and measured the projected velocity of the ejecta. We derived the spectral reflectance gradient (or colour) in the visible 
Table 7. Observed total dust mass ejected.

\begin{tabular}{|c|c|c|c|c|c|c|c|c|}
\hline \multirow[t]{2}{*}{ Img } & $\begin{array}{c}\text { Bulk } \\
\text { density } \\
\left(\mathrm{kg} \mathrm{m}^{-3}\right)\end{array}$ & $\begin{array}{c}\text { Range of } \\
\text { radius } \\
(\mu \mathrm{m})\end{array}$ & & & & (tons) & & \\
\hline \multirow{2}{*}{\multicolumn{9}{|c|}{$\begin{array}{l}-4.5 \\
2015 \text { Aug } 10\end{array}$}} \\
\hline & & & & & & & & \\
\hline A & 795 & $0.1-1000$ & 0.25 & 0.75 & 8.45 & 88.78 & 284.50 & 422.54 \\
\hline $\mathrm{C}$ & 795 & $0.1-1000$ & 0.19 & 0.58 & 6.43 & 67.54 & 216.44 & 321.45 \\
\hline $\mathrm{D}$ & 795 & $0.1-1000$ & 0.10 & 0.31 & 3.46 & 36.36 & 116.50 & 173.02 \\
\hline \multicolumn{9}{|c|}{2015 Sept 14} \\
\hline E & 795 & $0.1-1000$ & 0.03 & 0.08 & 0.95 & 10.00 & 32.00 & 47.54 \\
\hline $\mathrm{F}^{\star}$ & 795 & $0.1-1000$ & 0.33 & 1.01 & 11.34 & 119.17 & 381.89 & 567.16 \\
\hline $\mathrm{F}^{\star \star}$ & 795 & $0.1-1000$ & 0.43 & 1.31 & 14.74 & 154.92 & 496.46 & 737.31 \\
\hline
\end{tabular}

Notes. Img: Assigned letter for each image cube.

Bulk density: Particle bulk density.

Range of radius: Range of particle radius used for the calculations.

Total dust mass ejected: Observed total dust mass ejected computed for different differential size distribution indexes $(\alpha)$.

$\alpha$ : power-law index.

${ }^{\star}$ Ejected mass computed considering the outburst inside the FOV.

** Ejected mass computed considering an additional fraction of $30 \%$ of the outburst outside the FOV.

wavelengths and the total mass ejected during the outburst events. Our main results can be summarized as follows:

\section{Coma morphology and light curves}

- Several big or mini outbursts were detected on comet 67P/CG with maximum radiances ranging between 0.1 and $1.0 \mathrm{~W} \mathrm{~m}^{-2} \mathrm{sr}^{-1}$ $\mu \mathrm{m}^{-1}$ at $0.55 \mu \mathrm{m}$.

- The dust morphology of these outbursts can be classified into two main types: narrow and collimated plumes (August 10 and September 13) and broad blobs (September 14).

- The observations suggest that both big and mini outbursts have a tendency to trigger further outburst activity within a few minutes of the initial event, as evidenced by the September 13 and 14 outbursts.

- The temporal profiles of the outbursts are associated with a very rapid change in radiance from quiescent coma to the maximum observed, showing that the dust material is ejected from the nucleus in a short-lived event.

- The outburst durations observed in the VIS and IR channels range between 24 and $6 \mathrm{~min}$.

\section{Outburst particle dynamics}

- The dust ejecta from the big outbursts of 2015 September 13 and 14 expanded at speeds between $22.2 \pm 2.2$ and $30.2 \pm 1.4 \mathrm{~m} \mathrm{~s}^{-1}$. These values are similar to those found by other instruments onboard Rosetta. Agarwal et al. (2017) detected particles travelling at a speed of $(25 \pm 10) \mathrm{m} \mathrm{s}^{-1}$ at the beginning of the event. The velocities are similar to those found by Feldman et al. (2007) for Comet 9P/Tempel. These measured velocities are for small particles, which explain why they are higher than those measured by GIADA (Grün et al. 2016; Agarwal et al. 2017).

\section{Outburst location}

- The observations suggest that there are localized regions on the surface of the comet that are more prone to outbursts than the rest of the nucleus. All outburst sources are located in the Southern hemisphere of the main lobe, within the latitude range $-5--55^{\circ}$.

- The outbursts observed on 2015 September 14 have a broad morphology and a likely source location in the Imhotep region.
- The collimated events observed on 2015 August 10 and September 13 arise from the eastern side of the body around longitude $210-280^{\circ}$ and latitude $-20--55^{\circ}$, in areas characterized by steep scarps, cliffs, and pits with considerable talus deposits (El-Maarry et al. 2016; Pajola et al. 2016).

\section{Dust outburst properties}

- The outburst reflectance spectrum, in the range from 0.3 to $5.0 \mu \mathrm{m}$, can be fitted with two reflectance gradients with values of $12 \pm 0.6$ per cent $/ 100 \mathrm{~nm}$ in the VIS channel and $-2.1 \pm 0.1$ per cent/100 $\mathrm{nm}$ in the IR channel.

- The outburst colour maps in the VIS show a colour gradient from red to blue associated with the sudden increase of dust continuum radiance of the outbursts. The same colour behaviour has been observed in the IR channel reaching the bluer values of $-9.1 \pm 1.4$ per cent $/ 100 \mathrm{~nm}$ and returning to the pre-outburst value of about 2.5 per cent $/ 100 \mathrm{~nm}$. The IR continuum emission is also characterized by high colour temperatures of about $600 \mathrm{~K}$ and a bolometric albedo of 0.6. The combination of VIS and IR dust properties reveals the presence of very small grains (less than $100 \mathrm{~nm}$ ) in the outburst material. The bright grains in the ejecta could be silicate grains, implying the thermal degradation of the carbonaceous material, or icy grains.

- The far-ultraviolet spectrograph, Alice, onboard Rosetta has observed a strong absorption feature around $170 \mathrm{~nm}$, characteristic of water ice (Steffl et al. 2016). However, in the IR spectra, the water ice absorption band at $3 \mu \mathrm{m}$ is not detected. The presence of absorption feature around $170 \mathrm{~nm}$ and the absence at $3 \mu \mathrm{m}$ would be consistent with the presence of very small ice particles, less than $100 \mathrm{~nm}$ (Bockelée-Morvan et al. 2017). The UV wavelengths are more sensitive to the small particles with respect to the IR wavelengths. This fact can provide a constrain about the size of particles inside the outburst ejecta.

- The rapid increase in radiance at the start of an outburst event is not due primarily to an increase in the number of existing dust particles but to the release of small and bright icy particles with a high geometric albedo and a filling factor between 1.3 and 5.0 per cent. 


\section{Total dust mass ejected}

- Assuming a typical size distribution taken from the 67P/CG literature, with indexes between -2.5 and -3 , the observed total mass of ejected dust is estimated to be between 10 and 230 tons for a mini outburst and from 100 to 500 tons for the big outbursts.

\section{ACKNOWLEDGEMENTS}

We thank the following institutions and agencies for support of this work: Italian Space Agency (ASI, Italy) contract number I/024/12/1, Centre National d'Études Spatiales (CNES, France), DLR (Germany), NASA (USA) Rosetta Program, and Science and Technology Facilities Council (UK). VIRTIS was built by a consortium, which includes Italy, France, and Germany under the scientific responsibility of the Istituto di Astrofisica e Planetologia Spaziali of INAF, Italy, that also guides the scientific operations. The VIRTIS instrument development, led by the prime contractor LeonardoFinmeccanica (Florence, Italy), has been funded and managed by Agenzia Spaziale Italiana (ASI), with contributions from Observatoire de Meudon financed by (CNES)Centre National d'Études Spatiales, and from German Aerospace Center (DLR). We thank the Rosetta Science Ground Segment and the Rosetta Mission Operations Centre for their support throughout all the phases of the mission. The VIRTIS calibrated data will be available through the ESA's Planetary Science Archive website (http://www.rssd.esa.int) and is available upon request in advance of being posted to the archive.

GIADA was built by a consortium led by the Univ. Napoli Parthenope \& INAF- Oss. Astr. Capodimonte, in collaboration with the Inst. de Astrofisica de Andalucia, Selex-ES, FI and SENER. GIADA was managed \& operated at INAF-IAPS. GIADA was funded and managed by the Agenzia Spaziale Italiana, IT, with the support of the Spanish Ministry of Education and Science MEC, ES. GIADA calibrated data are available through ESA PSA website (http://www.rssd.esa.int/index.php?project=PSA\&page=index).

Additional computational resources used in this research have been partly supplied by INAF-IAPS through the DataWell project. The authors wish to thanks J.B. Vincent for his helpful suggestions and questions during the review process. Giovanna Rinaldi wishs to thanks Stefania Stefani and Francesca Napolitano for the important support given during the most critical phases of the works.

\section{REFERENCES}

A'Hearn M. F. et al., 2005, Science, 310, 258

Acton C. H., 1996, Planet. Space Sci., 44, 65

Agarwal J. et al., 2017, MNRAS, 469, s606

Ammannito E., Filacchione G., Coradini A., Capaccioni F., Piccioni G., de Sanctis M. C., Dami M., Barbis A., 2006, Rev. Sci. Instrum., 77, 093109 Belton M. J. S., Feldman P. D., A'Hearn M. F., Carcich B., 2008, Icarus, 198, 189
Bockelée-Morvan D. et al., 2017, MNRAS, 469, S443

Boissier J., Bockelée-Morvan D., Biver N., Crovisier J., Lellouch E., Moreno R., Zakharov V., 2012, A\&A, 542, A73

Capaccioni F. et al., 2015, Science, 347, aaa0628

Ciarniello M. et al., 2015, A\&A, 583, A31

Colangeli L. et al., 2007, Space Sci. Rev., 128, 803

Coradini A. et al., 2007, Space Sci. Rev., 128, 529

Della Corte V. et al., 2016, Acta Astronaut., 126, 205

Della Corte V., Rotundi A., Fulle M., Ivanovski S., Agarwal J., Gruen E., 2017, Eur. Planet. Sci. Congr., 11, EPSC2017

Drossart P. et al., 2000, in Strojnik M., Andresen B. F. eds, Proc. SPIE Conf. Ser. Vol. 4131, Infrared Spaceborne Remote Sensing VIII. SPIE, Bellingham, p. 78

El-Maarry M. R. et al., 2015, A\&A, 583, A26

El-Maarry M. R. et al., 2016, A\&A, 593, A110

El-Maarry M. R. et al., 2017, Science, 355, 1392

Farnham T. L. et al., 2007, Icarus, 187, 26

Feldman P. D., McCandliss S. R., Route M., Weaver H. A., A'Hearn M. F., Belton M. J. S., Meech K. J., 2007, Icarus, 187, 113

Filacchione G., Ammannito E., Coradini A., Capaccioni F., Piccioni G., de Sanctis M. C., Dami M., Barbis A., 2006, Rev. Sci. Instrum., 77, 103106 Fink U., Rinaldi G., 2015, Icarus, 257, 9

Fink U., Rubin M., 2012, Icarus, 221, 721

Fougere N. et al., 2016, MNRAS, 462, S156

Fulle M. et al., 2015, A\&A, 583, A14

Fulle M. et al., 2016, MNRAS, 462, S132

Fulle M. et al., 2017, MNRAS, 469, S45

Gicquel A. et al., 2017, MNRAS, 469, S178

Grün E. et al., 2016, MNRAS, 462, S220

Ivanovski S. L., Zakharov V. V., Della Corte V., Crifo J.-F., Rotundi A., Fulle M., 2017a, Icarus, 282, 333

Ivanovski S. L. et al., 2017b, MNRAS, 469, S774

Jewitt D., Luu J., 1990, ApJ, 365, 738

Jewitt D., Meech K. J., 1986, ApJ, 310, 937

Jorda L. et al., 2016, Icarus, 277, 257

Knollenberg J. et al., 2016, A\&A, 596, A89

Kramer T., Läuter M., Rubin M., Altwegg K., 2017, MNRAS, 469, S20

Kurucz R. L., 1994, in Rabin D. M., Jefferies J. T., Lindsey C. eds, Proc. IAU Symp. 154, Infrared Solar Physics, Kluwer, Dordrecht, p. 523

Oklay N. et al., 2016, A\&A, 586, A80

Pajola M. et al., 2016, A\&A, 592, A69

Pajola M. et al., 2017, Nature Astron., 1, 0092

Pätzold M. et al., 2016, Nature, 530, 63

Rinaldi G. et al., 2016, MNRAS, 462, S547

Rotundi A. et al., 2015, Science, 347, aaa3905

Sierks H. et al., 2015, Science, 347, aaa1044

Steffl A. J. et al., 2016, AAS/Division for Planetary Sciences Meeting Abstracts, p. 110.03

Tubiana C. et al., 2015, A\&A, 573, A62

Vincent J.-B. et al., 2016, MNRAS, 462, S184

Vincent J.-B. et al., 2017, MNRAS, 469, S329

This paper has been typeset from a $\mathrm{T}_{\mathrm{E}} \mathrm{X} / \mathrm{L} \mathrm{T} \mathrm{E} \mathrm{X}$ file prepared by the author. 